\title{
PREPRINT
}

\section{A nonlinear Klein-Gordon equation on a star graph}

\author{
Nataliia Goloshchapova
}

Institute of Mathematics, University of São

Paulo, São Paulo, Brazil

\section{Correspondence}

Email: nataliia@ime.usp.br

\section{Present Address}

R. do Matão, 1010, São Paulo - SP, 05508-090

\author{
Summary \\ We study local well-posedness and orbital stability/instability of standing waves for a \\ first order system associated with a nonlinear Klein-Gordon equation on a star graph. \\ The proof of the well-posedness uses a classical fixed point argument and the Hille- \\ Yosida theorem. Stability study relies on the linearization approach and recent results \\ for the NLS equation with the $\delta$-interaction on a star graph. \\ KEYWORDS: \\ $\delta$-interaction, nonlinear Klein-Gordon equation, orbital stability, standing wave, star graph.
}

\section{1 | INTRODUCTION}

The study of differential equations on graphs is a rapidly developing area (see [7] and the references therein). It is motivated by various physical applications involving wave propagation in narrow waveguides. Graphs arise as approximations of multidimensional narrow waveguides when their thickness parameters converge to zero. A large part of the literature is devoted to linear equations on graphs, with special emphasis on the Schrödinger equation describing the so-called quantum graphs. The models on a star graph $\Gamma$ constituted by $N$ half-lines joined at the vertex $v=0$ are one of the simplest. Recently a certain amount o research work has been done on the nonlinear Schrödinger equation with the $\delta$-interaction (NLS- $\delta$ ) on $\Gamma$ (see [4, 8, 18], and the references therein):

$$
i \partial_{t} \mathbf{u}(t, x)=H_{\alpha} \mathbf{u}(t, x)-|\mathbf{u}(t, x)|^{p-1} \mathbf{u}(t, x),
$$

where $p>1, \mathbf{u}(t, x)=\left(u_{j}(t, x)\right)_{j=1}^{N}: \mathbb{R} \times \mathbb{R}_{+} \rightarrow \mathbb{C}^{N}$, and $H_{\alpha}$ is the self-adjoint operator on $L^{2}(\Gamma)$ defined by

$$
\begin{aligned}
& \left(H_{\alpha} \mathbf{v}\right)(x)=\left(-v_{j}^{\prime \prime}(x)\right)_{j=1}^{N}, \quad x>0, \quad \mathbf{v}=\left(v_{j}\right)_{j=1}^{N}, \\
& \operatorname{dom}\left(H_{\alpha}\right)=\left\{\mathbf{v} \in H^{2}(\Gamma): v_{1}(0)=\ldots=v_{N}(0), \sum_{j=1}^{N} v_{j}^{\prime}(0)=\alpha v_{1}(0)\right\} .
\end{aligned}
$$

The NLS- $\delta$ equation has been studied in the context of well-posedness, variational properties, existence, and stability of standing waves. In his survey [18] about the NLS on graphs, Noja along with the model [1.1), mentioned (as one of the main examples of PDEs on the star graph) the following nonlinear Klein-Gordon equation with the $\delta$-interaction (NKG- $\delta$ ):

$$
-\partial_{t}^{2} \mathbf{u}(t, x)=H_{\alpha} \mathbf{u}(t, x)+m^{2} \mathbf{u}(t, x)-|\mathbf{u}(t, x)|^{p-1} \mathbf{u}(t, x) .
$$

On each edge of the graph (i.e. on each half-line) we have

$$
-\partial_{t}^{2} u_{j}(t, x)=-\partial_{x}^{2} u_{j}(t, x)+m^{2} u_{j}(t, x)-\left|u_{j}(t, x)\right|^{p-1} u_{j}(t, x), \quad x>0, \quad j \in\{1, \ldots, N\},
$$

moreover, the vectors $\mathbf{u}(t, 0)=\left(u_{j}(t, 0)\right)_{j=1}^{N}$ and $\mathbf{u}^{\prime}(t, 0)=\left(u_{j}^{\prime}(t, 0)\right)_{j=1}^{N}$ satisfy the conditions in (1.2). To our knowledge, the NKG- $\delta$ equation has never been studied in the context of well-posedness and stability of standing waves.

In the present paper we aim to initiate this study. The stability/instability study of standing wave solutions of the NKG equation in homogeneous media (in $n$ space dimensions) was started by Shatah in [23, 24], and then continued in [14, 19]. We rely on 
the recent research [9], where the authors considered the nonlinear Klein-Gordon equation with $\delta$-potentials on $\mathbb{R}$

$$
-\partial_{t}^{2} u(t, x)=-\partial_{x}^{2} u(t, x)+m^{2} u(t, x)+\gamma \delta(x) u(t, x)+i \alpha \delta(x) \partial_{t} u(t, x)-|u(t, x)|^{p-1} u(t, x),
$$

$\gamma, \alpha \in \mathbb{R}$, and $\delta(x)$ is Dirac delta function.

We prove local well-posedness of the Cauchy problem for a first order Hamiltonian system associated with (1.3), using classical approach related on the theory of $C_{0}$ semigroups (see [10, 20] for the detailed exposition). In particular, it has been shown that certain operator $A-\beta$ associated with equation (1.3) is dissipative.

The main goal is the study of orbital stability of the standing wave solutions $\mathbf{u}(t, x)=e^{i \omega t} \boldsymbol{\varphi ( x )}$ to (1.3), where the profile $\varphi(x)$ is a real-valued vector function. The profile $\varphi(x)$ satisfies the following stationary equation

$$
H_{\alpha} \varphi+\left(m^{2}-\omega^{2}\right) \boldsymbol{\varphi}-|\varphi|^{p-1} \boldsymbol{\varphi}=0 .
$$

Applying [1, Theorem 4], one gets the description of real-valued vector solutions to (1.4).

Theorem 1.1. Let $[s]$ denote the integer part of $s \in \mathbb{R}$, and $\alpha \neq 0$. Then equation (1.4) has $\left[\frac{N-1}{2}\right]+1$ (up to permutations of the edges of $\Gamma$ ) real-valued vector solutions $\varphi_{k, \omega}^{\alpha}=\left(\tilde{\varphi}_{k, j}\right)_{j=1}^{N}, k \in\left\{0, \ldots,\left[\frac{N-1}{2}\right]\right\}$, which are given by

$$
\begin{aligned}
& \tilde{\varphi}_{k, j}(x)= \begin{cases}{\left[\frac{(p+1)\left(m^{2}-\omega^{2}\right)}{2} \operatorname{sech}^{2}\left(\frac{(p-1) \sqrt{m^{2}-\omega^{2}}}{2} x-c_{k}\right)\right]^{\frac{1}{p-1}},} & j=1, \ldots, k ; \\
{\left[\frac{(p+1)\left(m^{2}-\omega^{2}\right)}{2} \operatorname{sech}^{2}\left(\frac{(p-1) \sqrt{\left(m^{2}-\omega^{2}\right)}}{2} x+c_{k}\right)\right]^{\frac{1}{p-1}},} & j=k+1, \ldots, N,\end{cases} \\
& \text { where } c_{k}=\tanh ^{-1}\left(\frac{\alpha}{(2 k-N) \sqrt{\left(m^{2}-\omega^{2}\right)}}\right), \text { and } m^{2}-\omega^{2}>\frac{\alpha^{2}}{(N-2 k)^{2}} .
\end{aligned}
$$

In Theorem 3.17 we provide a sufficient condition on the parameters $\omega, m, \alpha, k, N$ to get the orbital stability/instability of the standing waves $e^{i \omega t} \boldsymbol{\varphi}_{k, \omega}^{\alpha}(x)$. The orbital stability is studied in the context of a Hamiltonian system associated with the NKG- $\delta$ equation. Its investigation relies on the classical works by Grillakis, Shatah, and Strauss [12, 13] and recent work [25] by Stuart. The proof of stability/instability result essentially uses spectral analysis of certain self-adjoint Schrödinger operators on the star graph. This analysis was elaborated extensively in papers [3, 4] devoted to the stability study of standing waves for the NLS$\delta$ equation. The principal ingredients of the spectral analysis are the analytic perturbation theory and the extension theory of symmetric operators.

\section{Notation.}

Let $L$ be a densely defined symmetric operator in some Hilbert space. The deficiency numbers of $L$ are defined by $n_{ \pm}(L):=$ dim $\operatorname{ker}\left(L^{*} \mp i I\right)$. The number of negative eigenvalues counting multiplicities (the Morse index) is denoted by $n(L)$.

We regard $L^{2}\left(\mathbb{R}_{+}\right)$as a real Hilbert space with the inner product

$$
\langle u, v\rangle_{L^{2}\left(\mathbb{R}_{+}\right)}=\operatorname{Re} \int_{\mathbb{R}_{+}} u \bar{v} d x,
$$

and $H^{1}\left(\mathbb{R}_{+}\right)$as the Sobolev space with the inner product

$$
\langle u, v\rangle_{H^{1}\left(\mathbb{R}_{+}\right)}=\langle u, v\rangle_{L^{2}\left(\mathbb{R}_{+}\right)}+\left\langle u^{\prime}, v^{\prime}\right\rangle_{L^{2}\left(\mathbb{R}_{+}\right)} .
$$

We consider the star graph $\Gamma$ constituted by $N$ half-lines $\mathbb{R}_{+}$attached to a common vertex $v=0$. The function $\mathbf{w}$ acting on $\Gamma$ is represented by the vector $\left(w_{j}\right)_{j=1}^{N}$, where each scalar function $w_{j}$ is defined on $[0, \infty)$. For $\mathbf{w}=\left(w_{j}\right)_{j=1}^{N}$ on $\Gamma$, we will abbreviate

$$
\int_{\Gamma} \mathbf{w} d x=\sum_{j=1}^{N} \int_{\mathbb{R}_{+}} w_{j} d x
$$

On the graph we define the following spaces

$$
L^{q}(\Gamma)=\bigoplus_{j=1}^{N} L^{q}\left(\mathbb{R}_{+}\right), 1 \leq q \leq \infty, \quad H^{1}(\Gamma)=\bigoplus_{j=1}^{N} H^{1}\left(\mathbb{R}_{+}\right), \quad H^{2}(\Gamma)=\bigoplus_{j=1}^{N} H^{2}\left(\mathbb{R}_{+}\right) .
$$


The corresponding $L^{2}$ - and $H^{1}$-inner products are defined by

$$
\langle\mathbf{u}, \mathbf{v}\rangle_{L^{2}(\Gamma)}=\operatorname{Re} \int_{\Gamma} \mathbf{u} \overline{\mathbf{v}} d x, \quad\langle\mathbf{u}, \mathbf{v}\rangle_{H^{1}(\Gamma)}=\operatorname{Re}\left[\int_{\Gamma} \mathbf{u} \overline{\mathbf{v}} d x+\int_{\Gamma} \mathbf{u}^{\prime} \overline{\mathbf{v}}^{\prime} d x\right] .
$$

By $\mathcal{E}(\Gamma)$ we denote the space

$$
\mathcal{E}(\Gamma)=\left\{\mathbf{v} \in H^{1}(\Gamma): v_{1}(0)=\ldots=v_{N}(0)\right\}
$$

The dual space for $\mathcal{E}(\Gamma)$ is denoted by $\mathcal{E}^{*}(\Gamma)$.

Set $X=\mathcal{E}(\Gamma) \times L^{2}(\Gamma)=\left\{(\mathbf{u}, \mathbf{v}): \mathbf{u} \in \mathcal{E}(\Gamma), \mathbf{v} \in L^{2}(\Gamma)\right\}$ for the real Hilbert space with the inner product

$$
\left\langle\left(\mathbf{u}_{1}, \mathbf{v}_{1}\right),\left(\mathbf{u}_{2}, \mathbf{v}_{2}\right)\right\rangle_{X}=\left\langle\mathbf{u}_{1}, \mathbf{u}_{2}\right\rangle_{H^{1}(\Gamma)}+\left\langle\mathbf{v}_{1}, \mathbf{v}_{2}\right\rangle_{L^{2}(\Gamma)} .
$$

Its dual $X^{*}$ is identified with $\mathcal{E}^{*}(\Gamma) \times L^{2}(\Gamma)$, and the duality pairing is denoted by $\langle\cdot, \cdot\rangle_{X^{*} \times X}$. For $k \in\{0, \ldots, N-1\}$ we define the spaces

$$
\begin{aligned}
L_{k}^{2}(\Gamma) & =\left\{\mathbf{v} \in L^{2}(\Gamma): v_{1}(x)=\ldots=v_{k}(x), v_{k+1}(x)=\ldots=v_{N}(x), x \geq 0\right\}, \quad \text { and } \\
\mathcal{E}_{k}(\Gamma) & =\mathcal{E}(\Gamma) \cap L_{k}^{2}(\Gamma), \quad X_{k}=\mathcal{E}_{k}(\Gamma) \times L_{k}^{2}(\Gamma) .
\end{aligned}
$$

If $k=0$, then $L_{\mathrm{eq}}^{2}(\Gamma)=L_{0}^{2}(\Gamma), \mathcal{E}_{\mathrm{eq}}(\Gamma)=\mathcal{E}(\Gamma) \cap L_{\mathrm{eq}}^{2}(\Gamma)$, and $X_{\mathrm{eq}}=\mathcal{E}_{\mathrm{eq}}(\Gamma) \times L_{\mathrm{eq}}^{2}(\Gamma)$.

\section{2 | LOCAL WELL-POSEDNESS}

We consider the following Cauchy problem

$$
\left\{\begin{array}{l}
-\partial_{t}^{2} \mathbf{u}(t, x)=H_{\alpha} \mathbf{u}(t, x)+m^{2} \mathbf{u}(t, x)-|\mathbf{u}|^{p-1} \mathbf{u} \\
\mathbf{u}(0, x)=\mathbf{u}_{0}(x) \\
\boldsymbol{\partial}_{t} \mathbf{u}(0, x)=\mathbf{u}_{1}(x)
\end{array}\right.
$$

Let us reformulate (2.1) as a first order system on $X$. Denoting $\mathbf{v}=\partial_{t} \mathbf{u}, \mathbf{U}=(\mathbf{u}, \mathbf{v}), F(\mathbf{U})=\left(0,|\mathbf{u}|^{p-1} \mathbf{u}\right)$, and $\mathbf{U}_{0}=\left(\mathbf{u}_{0}, \mathbf{u}_{1}\right)$, we formally get from 2.1)

$$
\left\{\begin{array}{l}
\partial_{t} \mathbf{U}(t)=A \mathbf{U}(t)+F(\mathbf{U}(t)) \\
\mathbf{U}(0)=\mathbf{U}_{0}
\end{array}\right.
$$

where

$$
\begin{gathered}
A=\left(\begin{array}{cc}
0 & I d_{L^{2}(\Gamma)} \\
-H_{\alpha}-m^{2} & 0
\end{array}\right), \\
\operatorname{dom}(A)=\left\{\begin{array}{c}
(\mathbf{u}, \mathbf{v}) \in H^{2}(\Gamma) \times \mathcal{E}(\Gamma): \\
u_{1}(0)=\ldots=u_{N}(0), \sum_{j=1}^{N} u_{j}^{\prime}(0)=\alpha u_{1}(0)
\end{array}\right\}=\operatorname{dom}\left(H_{\alpha}\right) \times \mathcal{E}(\Gamma) .
\end{gathered}
$$

Below we will prove existence and uniqueness of a weak solution $\mathbf{U}(t) \in C([0, T], X)$ to system (2.2) (see [5] for the definition of a weak solution). The proof is in the spirit of [10, Chapter 4]. First, we prove that operator $A$ generates strongly continuous semigroup on $X$.

Proposition 2.1. The operator $A$ generates $C_{0}$-semigroup on $X$. Moreover, there exist $M \geq 0$ and $\beta \geq 0$ such that for all $t \geq 0$ the following estimate holds

$$
\left\|e^{t A}\right\| \leq M e^{\beta t}
$$

Proof. Our aim is to apply [20, Chapter I, Corollary 3.8]. We need to prove density of $\operatorname{dom}(A)$ in $X$.

Step 1. Let $(\mathbf{u}, \mathbf{v}) \in X$. Obviously there exists a sequence $\left\{\mathbf{v}_{n}\right\}_{n=1}^{\infty} \subset \mathcal{E}(\Gamma)$ such that $\mathbf{v}_{n} \underset{n \rightarrow \infty}{\rightarrow} \mathbf{v}$ in $L^{2}(\Gamma)$ (indeed, $\operatorname{dom}\left(H_{\alpha}\right) \subset$ $\mathcal{E}(\Gamma)$ and $\left.\overline{\operatorname{dom}\left(H_{\alpha}\right)}=L^{2}(\Gamma)\right)$. We need to show that there exists a sequence $\left\{\mathbf{u}_{n}\right\}_{n=1}^{\infty} \subset \operatorname{dom}\left(H_{\alpha}\right)$ such that $\mathbf{u}_{n} \underset{n \rightarrow \infty}{\rightarrow} \mathbf{u} \in \mathcal{E}(\Gamma)$ in 
$H^{1}(\Gamma)$. Consider the following self-adjoint operator $H_{0}$ in $L^{2}(\Gamma)$ (with the Kirchhoff conditions)

$$
\begin{aligned}
& \left(H_{0} \mathbf{w}\right)(x)=\left(-w_{j}^{\prime \prime}(x)\right)_{j=1}^{N}, \quad x>0, \quad \mathbf{w}=\left(w_{j}\right)_{j=1}^{N}, \\
& \operatorname{dom}\left(H_{0}\right)=\left\{\mathbf{w} \in H^{2}(\Gamma): w_{1}(0)=\ldots=w_{N}(0), \sum_{j=1}^{N} w_{j}^{\prime}(0)=0\right\} .
\end{aligned}
$$

We show that there exists a sequence $\left\{\tilde{\mathbf{u}}_{n}\right\}_{n=1}^{\infty} \subset \operatorname{dom}\left(H_{0}\right)$ such that $\tilde{\mathbf{u}}_{n} \underset{n \rightarrow \infty}{\rightarrow} \mathbf{u}$ in $H^{1}(\Gamma)$, that is, $\overline{\operatorname{dom}\left(H_{0}\right)}=\mathcal{E}(\Gamma)$. It is sufficient to show that orthogonal complement of $\operatorname{dom}\left(H_{0}\right)$ in $\mathcal{E}(\Gamma)$ is $\{0\}$.

Let $\mathbf{z} \in \operatorname{dom}\left(H_{0}\right)^{\perp}$ in $\mathcal{E}(\Gamma)$, hence for any $\mathbf{w} \in \operatorname{dom}\left(H_{0}\right)$

$$
\begin{aligned}
& \langle\mathbf{w}, \mathbf{z}\rangle_{H^{1}(\Gamma)}=\langle\mathbf{w}, \mathbf{z}\rangle_{L^{2}(\Gamma)}+\left\langle\mathbf{w}^{\prime}, \mathbf{z}^{\prime}\right\rangle_{L^{2}(\Gamma)} \\
& =\langle\mathbf{w}, \mathbf{z}\rangle_{L^{2}(\Gamma)}-\operatorname{Re}\left(\sum_{j=1}^{N} w_{j}^{\prime}(0) \bar{z}_{j}(0)\right)-\left\langle\mathbf{w}^{\prime \prime}, \mathbf{z}\right\rangle_{L^{2}(\Gamma)}=\left\langle-\mathbf{w}^{\prime \prime}+\mathbf{w}, \mathbf{z}\right\rangle_{L^{2}(\Gamma)}=0 .
\end{aligned}
$$

The last equality implies $\mathbf{z} \in \operatorname{ran}\left(H_{0}+1\right)^{\perp}=\operatorname{ker}\left(H_{0}+1\right)=\{0\}$.

We modify the sequence $\left\{\tilde{\mathbf{u}}_{n}\right\}_{n=1}^{\infty}$ to get another one $\left\{\mathbf{u}_{n}\right\}_{n=1}^{\infty} \subset \operatorname{dom}\left(H_{\alpha}\right)$ that approximates $\mathbf{u}$ in $H^{1}(\Gamma)$. Define the sequence $\left\{\zeta_{n}\right\}_{n=1}^{\infty}$ by $\zeta_{n}=\left(1+\frac{\alpha x}{N} e^{-n x^{2}}\right)_{j=1}^{N}$. Let us show that $\left\{\mathbf{u}_{n}\right\}_{n=1}^{\infty}:=\left\{\zeta_{n} \tilde{\mathbf{u}}_{n}\right\}_{n=1}^{\infty} \subset \operatorname{dom}\left(H_{\alpha}\right)$ and $\mathbf{u}_{n} \underset{n \rightarrow \infty}{\rightarrow} \mathbf{u}$ in $H^{1}(\Gamma)$. It is easily seen that

which yields

$$
u_{j n}(0)=\tilde{u}_{j n}(0), \quad u_{j n}^{\prime}(0)=\frac{\alpha}{N} \tilde{u}_{j n}(0)+\tilde{u}_{j n}^{\prime}(0)
$$

Therefore, $\left\{\mathbf{u}_{n}\right\}_{n=1}^{\infty} \subset \operatorname{dom}\left(H_{\alpha}\right)$. Observing that

$$
\sum_{j=1}^{N} u_{j n}^{\prime}(0)=\sum_{j=1}^{N}\left(\frac{\alpha}{N} \tilde{u}_{j n}(0)+\tilde{u}_{j n}^{\prime}(0)\right)=\alpha \tilde{u}_{1 n}(0)=\alpha u_{1 n}(0) .
$$

$$
\left\|\zeta_{n}-\mathbf{1}\right\|_{L^{\infty}(\Gamma)} \underset{n \rightarrow \infty}{\rightarrow} 0, \quad\left\|\zeta_{n}^{\prime}\right\|_{L^{\infty}(\Gamma)} \underset{n \rightarrow \infty}{\rightarrow} 0,
$$

we conclude $\left\|\mathbf{u}_{n}-\tilde{\mathbf{u}}_{n}\right\|_{H^{1}(\Gamma)} \underset{n \rightarrow \infty}{\rightarrow} 0$, consequently $\left\|\mathbf{u}_{n}-\mathbf{u}\right\|_{H^{1}(\Gamma)} \underset{n \rightarrow \infty}{\rightarrow} 0$. Finally, $\left\|\left(\mathbf{u}_{n}, \mathbf{v}_{n}\right)-(\mathbf{u}, \mathbf{v})\right\|_{X} \underset{n \rightarrow \infty}{\rightarrow} 0$.

Step 2. To prove inequality (3.21) on the resolvent $(A-\lambda)^{-1}$ in [20, Chapter I], we introduce alternative equivalent norm on $X$. It is known that $\inf \sigma\left(H_{\alpha}\right)=\left\{\begin{array}{c}0, \alpha \geq 0, \\ -\frac{\alpha^{2}}{N^{2}}, \alpha<0 .\end{array}\right.$ See, for example, Proposition 3.6 below for the proof of the identity $\sigma_{\text {ess }}\left(H_{\alpha}\right)=[0, \infty)$. The analysis of the discrete spectrum is trivial.

Given $\mu^{2}>-\inf \sigma\left(H_{\alpha}\right)$, then denoting

$$
\|\mathbf{u}\|_{1, \mu}^{2}:=\left\|\mathbf{u}^{\prime}\right\|_{L^{2}(\Gamma)}^{2}+\mu^{2}\|\mathbf{u}\|_{L^{2}(\Gamma)}^{2}+\alpha\left|u_{1}(0)\right|^{2},
$$

by the Sobolev embedding and inequality

$$
\left\|\mathbf{u}^{\prime}\right\|_{L^{2}(\Gamma)}^{2}+\alpha\left|u_{1}(0)\right|^{2} \geq \inf \sigma\left(H_{\alpha}\right)\|\mathbf{u}\|_{L^{2}(\Gamma)},
$$

we get

Therefore, the quadratic form defined on $X$ by

$$
C_{1}\|\mathbf{u}\|_{H^{1}(\Gamma)} \leq\|\mathbf{u}\|_{1, \mu} \leq C_{2}\|\mathbf{u}\|_{H^{1}(\Gamma)} .
$$

$$
\|(\mathbf{u}, \mathbf{v})\|_{X, \mu}^{2}=\|\mathbf{u}\|_{1, \mu}^{2}+\|\mathbf{v}\|_{L^{2}(\Gamma)}^{2}
$$

gives a norm on $X$ equivalent to $\|\cdot\|_{X}$. The corresponding inner product is given by

$$
\left\langle\left(\mathbf{u}_{1}, \mathbf{v}_{1}\right),\left(\mathbf{u}_{2}, \mathbf{v}_{2}\right)\right\rangle_{X, \mu}=\left\langle\mathbf{u}_{1}^{\prime}, \mathbf{u}_{2}^{\prime}\right\rangle_{L^{2}(\Gamma)}+\mu^{2}\left\langle\mathbf{u}_{1}, \mathbf{u}_{2}\right\rangle_{L^{2}(\Gamma)}+\alpha \operatorname{Re}\left(u_{11}(0) \bar{u}_{12}(0)\right)+\left\langle\mathbf{v}_{1}, \mathbf{v}_{2}\right\rangle_{L^{2}(\Gamma)} .
$$

Step 3. Suppose that $\mu$ is such that $\mu^{2}>m^{2}$. Let $\mathbf{U}=(\mathbf{u}, \mathbf{v}) \in \operatorname{dom}(A)$, then

$$
\begin{aligned}
& \langle A \mathbf{U}, \mathbf{U}\rangle_{X, \mu}=\left\langle\mathbf{v}^{\prime}, \mathbf{u}^{\prime}\right\rangle_{L^{2}(\Gamma)}+\mu^{2}\langle\mathbf{v}, \mathbf{u}\rangle_{L^{2}(\Gamma)}+\alpha \operatorname{Re}\left(v_{1}(0) \bar{u}_{1}(0)\right)+\left\langle\mathbf{u}^{\prime \prime}, \mathbf{v}\right\rangle_{L^{2}(\Gamma)}-m^{2}\langle\mathbf{u}, \mathbf{v}\rangle_{L^{2}(\Gamma)} \\
& =\left\langle\mathbf{v}^{\prime}, \mathbf{u}^{\prime}\right\rangle_{L^{2}(\Gamma)}+\left(\mu^{2}-m^{2}\right)\langle\mathbf{v}, \mathbf{u}\rangle_{L^{2}(\Gamma)}+\alpha \operatorname{Re}\left(v_{1}(0) \bar{u}_{1}(0)\right)-\left\langle\mathbf{u}^{\prime}, \mathbf{v}^{\prime}\right\rangle_{L^{2}(\Gamma)}-\alpha \operatorname{Re}\left(u_{1}(0) \bar{v}_{1}(0)\right) \\
& =\left(\mu^{2}-m^{2}\right)\langle\mathbf{v}, \mathbf{u}\rangle_{L^{2}(\Gamma)} .
\end{aligned}
$$

Hence

$$
\left|\langle A \mathbf{U}, \mathbf{U}\rangle_{X, \mu}\right|=\left(\mu^{2}-m^{2}\right)\left|\langle\mathbf{v}, \mathbf{u}\rangle_{L^{2}(\Gamma)}\right| \leq \frac{\mu^{2}-m^{2}}{2}\left(\|\mathbf{u}\|_{L^{2}(\Gamma)}^{2}+\|\mathbf{v}\|_{L^{2}(\Gamma)}^{2}\right) .
$$


Observing that

$$
\|\mathbf{U}\|_{X, \mu}^{2}=\|\mathbf{u}\|_{1, \mu}^{2}+\|\mathbf{v}\|_{L^{2}(\Gamma)}^{2} \geq C_{1}^{2}\|\mathbf{u}\|_{H^{1}(\Gamma)}^{2}+\|\mathbf{v}\|_{L^{2}(\Gamma)}^{2} \geq C\left(\|\mathbf{u}\|_{L^{2}(\Gamma)}^{2}+\|\mathbf{v}\|_{L^{2}(\Gamma)}^{2}\right),
$$

for $\beta \geq 0$ large enough one gets

$$
\langle(A-\beta) \mathbf{U}, \mathbf{U}\rangle_{X, \mu} \leq 0 .
$$

Therefore, by [10, Proposition 2.4.2], the operator $A-\beta$ is dissipative. By dissipativity, for $\lambda>\beta$ one easily gets

$$
\begin{aligned}
\|(A-\lambda) \mathbf{U}\|_{X, \mu}^{2}=2(\beta-\lambda)\langle(A-\beta) \mathbf{U}, \mathbf{U}\rangle_{X, \mu} & +\|(A-\beta) \mathbf{U}\|_{X, \mu}^{2}+(\lambda-\beta)^{2}\|\mathbf{U}\|_{X, \mu}^{2} \\
& \geq\|(A-\beta) \mathbf{U}\|_{X, \mu}^{2}+(\lambda-\beta)^{2}\|\mathbf{U}\|_{X, \mu}^{2} .
\end{aligned}
$$

The above inequality implies that $\operatorname{ker}(A-\lambda)=\{0\}$. We show that $A-\lambda$ is surjective, i.e. $\operatorname{ran}(A-\lambda)=X$. Let $\mathbf{F}=(\mathbf{f}, \mathbf{g}) \in X$.

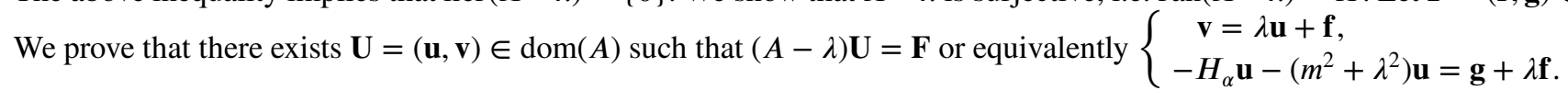

It is obvious that for $\lambda>\beta>\sqrt{\mu^{2}-m^{2}}$ the equation $-H_{\alpha} \mathbf{u}-\left(m^{2}+\lambda^{2}\right) \mathbf{u}=\mathbf{g}+\lambda \mathbf{f}$ has a unique solution $\mathbf{u}=-\left(H_{\alpha}+m^{2}+\right.$ $\left.\lambda^{2}\right)^{-1}(\mathbf{g}+\lambda \mathbf{f})$ (indeed, $\left.-\lambda^{2}-m^{2} \in \rho\left(H_{\alpha}\right)\right)$, and therefore $\mathbf{v}=-\lambda\left(H_{\alpha}+m^{2}+\lambda^{2}\right)^{-1}(\mathbf{g}+\lambda \mathbf{f})+\mathbf{f}$. This implies $\operatorname{ran}(A-\lambda)=X$, and finally, by estimate (2.6), the operator $A-\lambda$ has a bounded everywhere defined inverse, i.e.

$$
\left\|(A-\lambda)^{-1}\right\| \leq \frac{1}{\lambda-\beta} .
$$

In particular, for $\beta$ large enough $(\beta, \infty) \subset \rho(A)$, and hence $A$ is closed. Recalling that $\overline{\operatorname{dom}(A)}=X$, by corollary of the HilleYosida theorem (see [20, Chapter I, Corollary 3.8]), $A$ generates a $C_{0} \operatorname{semigroup~on~}\left(X,\|\cdot\|_{X, \mu}\right)$. Moreover, for $t \geq 0$ and $\mathbf{U} \in X$, we have

$$
\left\|e^{t A} \mathbf{U}\right\|_{X, \mu} \leq e^{\beta t}\|\mathbf{U}\|_{X, \mu} .
$$

By equivalence of the norms, there exists $M>0$ such that

$$
\left\|e^{t A} \mathbf{U}\right\|_{X} \leq M e^{\beta t}\|\mathbf{U}\|_{X}
$$

Remark 2.2. Observe that analogously one might show that for $\beta$ large enough $(\beta, \infty) \subset \rho(-A)$ and $\left\|(-A-\lambda)^{-1}\right\| \leq \frac{1}{\lambda-\beta}$, and therefore

$$
\left\|e^{-t A}\right\| \leq M e^{\beta t}, \quad t \geq 0, \quad \Longleftrightarrow \quad\left\|e^{t A}\right\| \leq M e^{-\beta t}, \quad t \leq 0 .
$$

Hence

$$
\left\|e^{t A}\right\| \leq M e^{\beta|t|}, \quad t \in \mathbb{R} .
$$

Remark 2.3. The fact that $\overline{\operatorname{dom}\left(H_{\alpha}\right)}=\mathcal{E}(\Gamma)$ can be shown alternatively applying the Representation Theorem [16, Chapter VI, Theorem 2.1]. Namely, one can use that $\operatorname{dom}\left(H_{\alpha}\right)$ is a core of the form

$$
t_{\alpha}(\mathbf{u}, \mathbf{v})=\left\langle\mathbf{u}^{\prime}, \mathbf{v}^{\prime}\right\rangle_{L^{2}(\Gamma)}+\alpha \operatorname{Re}\left(u_{1}(0) \overline{v_{1}}(0)\right), \quad \operatorname{dom}\left(t_{\alpha}\right)=\mathcal{E}(\Gamma) .
$$

Using Proposition 2.1, we obtain the following well-posedness theorem.

Theorem 2.4. Let $p>1$. Then

(i) for any $\mathbf{U}_{0}=\left(\mathbf{u}_{0}, \mathbf{u}_{1}\right) \in X$ there exists $T>0$ such that problem (2.2) has a unique weak solution $\mathbf{U}(t)=(\mathbf{u}(t), \mathbf{v}(t)) \in$ $C([0, T], X)$;

(ii) problem (2.2) has a maximal solution defined on an interval of the form [0, $T_{\max }$ ), and the following blow-up alternative holds: either $T_{\max }=\infty$ or $T_{\max }<\infty$ and

$$
\lim _{t \rightarrow T_{\max }}\|\mathbf{U}(t)\|_{X}=\infty
$$

(iii) for each $T \in\left(0, T_{\max }\right)$ the mapping $\mathbf{U}_{0} \in X \mapsto \mathbf{U}(t) \in C([0, T], X)$ is continuous;

(iv) the solution $\mathbf{U}(t)$ satisfies conservation of charge and energy:

$$
E(\mathbf{U}(t))=E\left(\mathbf{U}_{0}\right), \quad Q(\mathbf{U}(t))=Q\left(\mathbf{U}_{0}\right) \text { for all } t \in\left[0, T_{\max }\right),
$$


where

$$
\begin{aligned}
& E(\mathbf{u}, \mathbf{v})=\frac{1}{2}\left\|\mathbf{u}^{\prime}\right\|_{L^{2}(\Gamma)}^{2}+\frac{\alpha}{2}\left|u_{1}(0)\right|^{2}+\frac{m^{2}}{2}\|\mathbf{u}\|_{L^{2}(\Gamma)}^{2}-\frac{1}{p+1}\|\mathbf{u}\|_{L^{p+1}(\Gamma)}^{p+1}+\frac{1}{2}\|\mathbf{v}\|_{L^{2}(\Gamma)}^{2}, \\
& Q(\mathbf{u}, \mathbf{v})=\operatorname{Im} \int_{\Gamma} \mathbf{u} \overline{\mathbf{v}} d x .
\end{aligned}
$$

Proof. (i) Firstly, we prove that the nonlinearity $F$ is Lipschitz continuous on bounded sets of $X$, i.e.

$$
\|F(\mathbf{U})-F(\mathbf{W})\|_{X} \leq C(R)\|\mathbf{U}-\mathbf{W}\|_{X},
$$

for $\mathbf{U}, \mathbf{W} \in X, R>0$ with $\|\mathbf{U}\|_{X} \leq R,\|\mathbf{W}\|_{X} \leq R$.

For $\mathbf{u}=\left(u_{j}\right)_{j=1}^{N}$ and $\mathbf{w}=\left(w_{j}\right)_{j=1}^{N}$ one has

$$
\|\left. u_{j}\right|^{p-1} u_{j}-\left|w_{j}\right|^{p-1} w_{j}\left|\leq C\left(\left|u_{j}\right|^{p-1}+\left|w_{j}\right|^{p-1}\right)\right| u_{j}-w_{j} \mid
$$

which implies

$$
\left\||\mathbf{u}|^{p-1} \mathbf{u}-|\mathbf{w}|^{p-1} \mathbf{w}\right\|_{L^{2}(\Gamma)} \leq C_{1}\left(\|\mathbf{u}\|_{L^{\infty}(\Gamma)}^{p-1}+\|\mathbf{w}\|_{L^{\infty}(\Gamma)}^{p-1}\right)\|\mathbf{u}-\mathbf{w}\|_{L^{2}(\Gamma)} .
$$

Therefore, by the Gagliardo-Nirenberg inequality (see formula (2.3) in [1]),

$$
\|\boldsymbol{\Psi}\|_{L^{p}(\Gamma)} \leq C\left\|\boldsymbol{\Psi}^{\prime}\right\|_{L^{2}(\Gamma)}^{\frac{1}{2}-\frac{1}{p}}\|\Psi\|_{L^{2}(\Gamma)}^{\frac{1}{2}+\frac{1}{p}}, p \in[2, \infty], \Psi \in H^{1}(\Gamma),
$$

we have

$$
\left\||\mathbf{u}|^{p-1} \mathbf{u}-|\mathbf{w}|^{p-1} \mathbf{w}\right\|_{L^{2}(\Gamma)} \leq C(R)\|\mathbf{u}-\mathbf{w}\|_{L^{2}(\Gamma)} .
$$

Hence for $\mathbf{U}=(\mathbf{u}, \mathbf{v}), \mathbf{W}=(\mathbf{w}, \mathbf{z}) \in X$

$$
\|F(\mathbf{U})-F(\mathbf{W})\|_{X}=\left\||\mathbf{u}|^{p-1} \mathbf{u}-|\mathbf{w}|^{p-1} \mathbf{w}\right\|_{L^{2}(\Gamma)} \leq C(R)\|\mathbf{u}-\mathbf{w}\|_{L^{2}(\Gamma)} \leq C(R)\|\mathbf{U}-\mathbf{W}\|_{X} .
$$

Secondly, we show the existence of the solution. Now let $R$ and $T$ be two positive constants to be defined later. Consider the set

$$
X_{R}:=\left\{\mathbf{U}(t) \in C([0, T], X):\|\mathbf{U}(t)\|_{X} \leq R\right\}
$$

and the metric

$$
d(\mathbf{U}, \mathbf{V}):=\max _{t \in[0, T]}\|\mathbf{U}(t)-\mathbf{V}(t)\|_{X} .
$$

Observe that $\left(X_{R}, d\right)$ is a complete metric space. Now we define the map

$$
\mathcal{H}(\mathbf{U})(t)=e^{t A} \mathbf{U}_{0}+\int_{0}^{t} e^{(t-s) A} F(\mathbf{U}(s)) d s .
$$

It is obvious that $\mathcal{H}: X_{R} \mapsto C([0, T], X)$. We choose $T$ in order to guarantee invariance of $X_{R}$ for the mapping $\mathcal{H}$. By (2.3) and (2.7), we get

$$
\|\mathcal{H}(\mathbf{U})(t)\|_{X} \leq\left\|e^{t A} \mathbf{U}_{0}\right\|_{X}+\int_{0}^{t}\left\|e^{(t-s) A} F(\mathbf{U}(s))\right\|_{X} d s \leq M e^{\beta T}\left\|\mathbf{U}_{0}\right\|_{X}+T C(R) M e^{\beta T} R .
$$

Let $\frac{R}{4}=M\left\|\mathbf{U}_{0}\right\|_{X}$. By choosing $T$ small enough (for example, take $T \leq \min \left\{\frac{\ln 2}{\beta}, \frac{1}{4 C(R) M}\right\}$ ), we get

$$
\|\mathcal{H}(\mathbf{U})(t)\|_{X} \leq R
$$

And finally, we need to choose $T$ to get the contraction property of $\mathcal{H}$. For $\mathbf{U}, \mathbf{V} \in X_{R}$ one has

$$
\|\mathcal{H}(\mathbf{U})(t)-\mathcal{H}(\mathbf{V})(t)\|_{X} \leq \int_{0}^{t}\left\|e^{(t-s) A}(F(\mathbf{U}(s))-F(\mathbf{V}(s)))\right\|_{X} d s \leq M e^{T \beta} C(R) T d(\mathbf{U}, \mathbf{V}) .
$$

It is easily seen that $T$ can be chosen small enough to satisfy $M e^{T \beta} C(R) T<1$. Thus, the existence of the solution is established by the Banach fixed point theorem. 
Thirdly, we prove the uniqueness of the solution. It follows from Gronwall's lemma. Indeed, suppose that $\mathbf{U}_{1}, \mathbf{U}_{2} \in$ $C([0, T], X)$ are two solutions and $\left\|\mathbf{U}_{j}(t)\right\|_{X} \leq K, j \in\{1,2\}, t \in[0, T]$. Then

$$
\left\|\mathbf{U}_{1}(t)-\mathbf{U}_{2}(t)\right\|_{X} \leq \int_{0}^{t}\left\|e^{(t-s) A}\left(F\left(\mathbf{U}_{1}(s)\right)-F\left(\mathbf{U}_{2}(s)\right)\right)\right\|_{X} d s \leq M e^{T \beta} C(K) \int_{0}^{t}\left\|\mathbf{U}_{1}(s)-\mathbf{U}_{2}(s)\right\|_{X} d s,
$$

hence $\mathbf{U}_{1}(t)=\mathbf{U}_{2}(t), t \in[0, T]$.

(ii) The blow-up alternative follows by a bootstrap argument (see [10, Theorem 4.3.4]).

(iii) Repeating the proof of [10, Proposition 4.3.7], we can show lower semicontinuity of $T: X \rightarrow(0, \infty]$ and continuous dependence: if $\mathbf{U}_{0}^{n} \underset{n \rightarrow \infty}{\rightarrow} \mathbf{U}_{0}$ in $X$ and $T<T_{\max }$, then $\mathbf{U}^{n}(t) \underset{n \rightarrow \infty}{\rightarrow} \mathbf{U}(t)$ in $C([0, T], X)$.

(iv) Finally, we show the conservation laws. Firstly, observe that, using [10, Corollary 1.4.41] and [10, Proposition 4.1.6], one can prove the regularity property: for $\mathbf{U}_{0} \in \operatorname{dom}(A)$, there exists $T>0$ such that problem (2.2) has a unique solution $\mathbf{U}(t) \in C([0, T], \operatorname{dom}(A)) \cap C^{1}([0, T], X)$ (see also [10, Proposition 4.3.9]). Secondly, let us prove that the conservation of charge and energy hold for the solution $\mathbf{U}(t)=\left(\mathbf{u}(t), \partial_{t} \mathbf{u}(t)\right)$ with $\mathbf{U}_{0} \in \operatorname{dom}(A)$. Using the regularity property, one shows that

$$
\begin{aligned}
& \frac{d}{d t} Q(\mathbf{U}(t))=\operatorname{Im} \int_{\Gamma} \overline{\mathbf{u}} \overline{\partial_{t}^{2} \mathbf{u}} d x, \\
& \frac{d}{d t} E(\mathbf{U}(t))=\left\langle\partial_{t} \mathbf{u}, \partial_{t}^{2} \mathbf{u}\right\rangle_{L^{2}(\Gamma)}+\left\langle H_{\alpha} \mathbf{u}+m^{2} \mathbf{u}-|\mathbf{u}|^{p-1} \mathbf{u}, \partial_{t} \mathbf{u}\right\rangle_{L^{2}(\Gamma)}
\end{aligned}
$$

From (1.3) we get

$$
\frac{d}{d t} Q(\mathbf{U}(t))=\operatorname{Im} \int_{\Gamma} \overline{\mathbf{u}} \overline{\partial_{t}^{2} \mathbf{u}} d x=\operatorname{Im}\left[-\int_{\Gamma} \overline{\mathbf{u}} \overline{H_{\alpha} \mathbf{u}} d x-m^{2} \int_{\Gamma} \mathbf{u} \overline{\mathbf{u}} d x+\int_{\Gamma} \overline{\mathbf{u} g(\mathbf{u})} d x\right]=0,
$$

hence the charge is conserved.

Multiplying (2.2) by $\left(\overline{\partial_{t}^{2} \mathbf{u}},-\overline{\partial_{t} \mathbf{u}}\right)$ and integrating over $\Gamma$, we obtain

$$
0=\left\langle\partial_{t} \mathbf{u}, \partial_{t}^{2} \mathbf{u}\right\rangle_{L^{2}(\Gamma)}-\left\langle\partial_{t}^{2} \mathbf{u}, \partial_{t} \mathbf{u}\right\rangle_{L^{2}(\Gamma)}=\left\langle\partial_{t} \mathbf{u}, \partial_{t}^{2} \mathbf{u}\right\rangle_{L^{2}(\Gamma)}+\left\langle H_{\alpha} \mathbf{u}+m^{2} \mathbf{u}-|\mathbf{u}|^{p-1} \mathbf{u}, \partial_{t} \mathbf{u}\right\rangle_{L^{2}(\Gamma)},
$$

therefore, the energy is conserved.

Consider now $\mathbf{U}_{0} \in X$, then there exists a unique solution $\mathbf{U}(t) \in C([0, T], X)$. Take $\left\{\mathbf{U}_{0}^{n}\right\}_{n=1}^{\infty} \subset \operatorname{dom}(A)$ such that $\mathbf{U}_{0}^{n} \underset{n \rightarrow \infty}{\rightarrow} \mathbf{U}_{0}$ in $X$. By the regularity property,

$$
\mathbf{U}^{n}(t) \in C\left(\left[0, T^{n}\right], \operatorname{dom}(A)\right) \cap C^{1}\left(\left[0, T^{n}\right], X\right), \quad T^{n}<T_{\max }^{n} .
$$

For each $\mathbf{U}^{n}(t)$ the conservation laws hold:

$$
E\left(\mathbf{U}^{n}(t)\right)=E\left(\mathbf{U}_{0}^{n}\right), \quad Q\left(\mathbf{U}^{n}(t)\right)=Q\left(\mathbf{U}_{0}^{n}\right), \quad t \in\left[0, T_{\max }^{n}\right) .
$$

By continuous dependence and lower semicontinuity of $T$ we have that $\mathbf{U}^{n}(t) \underset{n \rightarrow \infty}{\rightarrow} \mathbf{U}(t)$ in $X$ for any $0 \leq t \leq T<T_{\max }^{n}$ (as $n$ is sufficiently large). Passing to the limit in (2.8), we obtain the result.

Remark 2.5. It is interesting to note that the conservation laws might be alternatively proved using $[11$, Theorem 6.8]. We need to show that the triple $(X, \operatorname{dom}(A), \mathcal{J})$, where

$$
\mathcal{J}: X \rightarrow X^{*}, \quad \mathcal{J}(\mathbf{u}, \mathbf{v})=(-\mathbf{v}, \mathbf{u})
$$

is a symplectic Banach triple (see [11, Definition 6.5]). It is easily seen that $\mathcal{J}$ is a symplector. In order to apply [11, Theorem 6.8] we need to prove that $E, Q \in \operatorname{Dif}(\operatorname{dom}(A), \mathcal{J})$. It means that $E$ and $Q$ have to be differentiable on $\operatorname{dom}(A)$ and $E^{\prime}(\mathbf{u}, \mathbf{v}), Q^{\prime}(\mathbf{u}, \mathbf{v})$ have to belong to $\operatorname{ran}(\mathcal{J})$ for any $(\mathbf{u}, \mathbf{v}) \in \operatorname{dom}(A)$. A simple check shows that for $(\mathbf{u}, \mathbf{v}) \in \operatorname{dom}(A)$ one gets

$$
E^{\prime}(\mathbf{u}, \mathbf{v})=\left(-\mathbf{u}^{\prime \prime}+m^{2} \mathbf{u}-|\mathbf{u}|^{p-1} \mathbf{u}, \mathbf{v}\right), \quad Q^{\prime}(\mathbf{u}, \mathbf{v})=(i \mathbf{v},-i \mathbf{u}),
$$

and obviously $E^{\prime}(\mathbf{u}, \mathbf{v}), Q^{\prime}(\mathbf{u}, \mathbf{v}) \in \operatorname{ran}(\mathcal{J})$.

To conclude the proof of the conservation laws we need to observe that $\{E, E\}(\mathbf{u}, \mathbf{v})=\{Q, E\}(\mathbf{u}, \mathbf{v})=0$ for all $(\mathbf{u}, \mathbf{v}) \in$ $\operatorname{dom}(A)$, where $\{\cdot, \cdot\}$ is the Poisson bracket defined by

$$
\{E, E\}(\mathbf{u}, \mathbf{v})=\left\langle E^{\prime}(\mathbf{u}, \mathbf{v}), \mathcal{J}^{-1} E^{\prime}(\mathbf{u}, \mathbf{v})\right\rangle_{X^{*} \times X}, \quad\{Q, E\}(\mathbf{u}, \mathbf{v})=\left\langle Q^{\prime}(\mathbf{u}, \mathbf{v}), \mathcal{J}^{-1} E^{\prime}(\mathbf{u}, \mathbf{v})\right\rangle_{X^{*} \times X} .
$$


Remark 2.6. Using definition of $\mathcal{J}$ and $E$, we can reformulate the system $\partial_{t} \mathbf{U}(t)=A \mathbf{U}(t)+F(\mathbf{U}(t))$ in the Hamiltonian form

$$
\mathcal{J} \partial_{t} \mathbf{U}(t)=E^{\prime}(\mathbf{U}(t))
$$

We finish this section by proving that problem (2.2) is well-posed in $X_{k}$.

Lemma 2.7. Let $k \in\{0, \ldots, N-1\}$. For any $\mathbf{U}_{0}=\left(\mathbf{u}_{0}, \mathbf{u}_{1}\right) \in X_{k}$ there exists $T>0$ such that (2.2) has a unique solution $\mathbf{U}(t)=(\mathbf{u}(t), \mathbf{v}(t)) \in C\left([0, T], X_{k}\right)$.

Proof. It is sufficient to prove that the corresponding $C_{0}$ semigroup $e^{t A}$ preserves the space $X_{k}$, that is, $e^{t A} X_{k} \subseteq X_{k}$. Equivalently this fact means that the solution to the Cauchy problem

$$
\left\{\begin{array}{l}
\partial_{t} \mathbf{U}(t)=A \mathbf{U}(t) \\
\mathbf{U}(0)=\mathbf{U}_{0}
\end{array}\right.
$$

belongs to $C\left([0, T], X_{k}\right)$ for $\mathbf{U}_{0} \in X_{k}$. Suppose that $k \geq 2$. Let

$$
\mathbf{U}(t)=e^{t A} \mathbf{U}_{0}=\left(\left(u_{j}(t)\right)_{j=1}^{N},\left(v_{j}(t)\right)_{j=1}^{N}\right)=\left(\left(u_{1}(t), \ldots, u_{N}(t)\right),\left(v_{1}(t), \ldots, v_{N}(t)\right)\right)
$$

be a solution to (2.11). Then the function

$$
\mathbf{V}(t)=\left(\left(u_{2}(t), u_{1}(t), u_{3}(t), \ldots, u_{N}(t)\right),\left(v_{2}(t), v_{1}(t), v_{3}(t) \ldots, v_{N}(t)\right)\right)
$$

is a solution to (2.11) as well. Indeed, the linear equation in (2.11) is invariant under the transposition of two first elements of the vector solution $\mathbf{U}(t)$. By uniqueness $\mathbf{U}(t)=\mathbf{V}(t)$, therefore, $u_{1}(t)=u_{2}(t)$ and $v_{1}(t)=v_{2}(t)$. Repeating the process, one gets $u_{1}(t)=\ldots=u_{k}(t), v_{1}(t)=\ldots=v_{k}(t)$.

Remark 2.8. The invariance property $e^{t A} X_{k} \subseteq X_{k}$ might be alternatively shown by involving functional calculus.

By [20, Chapter I, Corollary 7.5], for $\mathbf{W} \in \operatorname{dom}\left(A^{2}\right)$ we have

$$
e^{t A} \mathbf{W}=\frac{-1}{2 \pi i} \int_{\gamma-i \infty}^{\gamma+i \infty} e^{\lambda t}(A-\lambda)^{-1} \mathbf{W} d \lambda
$$

where $\gamma>\beta$ with $\beta$ from (2.3). Let $\lambda \in(\beta, \infty)$. We have

$$
A-\lambda=\left(\begin{array}{cc}
-\lambda I d_{\mathcal{E}(\Gamma)} & I d_{L^{2}(\Gamma)} \\
-H_{\alpha}-m^{2} & -\lambda I d_{L^{2}(\Gamma)}
\end{array}\right) .
$$

By a direct computation with operator-valued matrices,

$$
(A-\lambda)^{-1}=\left(\begin{array}{cc}
-\lambda\left(H_{\alpha}+\lambda^{2}+m^{2}\right)^{-1} & -\left(H_{\alpha}+\lambda^{2}+m^{2}\right)^{-1} \\
\left(H_{\alpha}+m^{2}\right)\left(H_{\alpha}+\lambda^{2}+m^{2}\right)^{-1} & -\lambda\left(H_{\alpha}+\lambda^{2}+m^{2}\right)^{-1}
\end{array}\right) .
$$

Observe that for $\beta$ large enough $-\lambda^{2} \in \rho\left(H_{\alpha}+m^{2}\right)$. Using formula (17) in [6] and denoting $z=\sqrt{m^{2}+\lambda^{2}}$, we get

$$
\left(\left(H_{\alpha}+\lambda^{2}+m^{2}\right)^{-1} \mathbf{w}\right)_{j}=\tilde{c}_{j} e^{-z x}+\frac{1}{2 z} \int_{0}^{\infty} w_{j}(y) e^{-|x-y| z} d y .
$$

Analogously to [4, Lemma 2.3], if $\mathbf{w} \in L_{k}^{2}(\Gamma)$, then $\tilde{c}_{1}=\ldots=\tilde{c}_{k}$ and $\tilde{c}_{k+1}=\ldots=\tilde{c}_{N}$, consequently $\left(H_{\alpha}+\lambda^{2}+m^{2}\right)^{-1} \mathbf{w} \in L_{k}^{2}(\Gamma)$. Hence, by (2.13), $(A-\lambda)^{-1} \mathbf{W} \in \operatorname{dom}(A) \cap X_{k}$ for $\mathbf{W} \in X_{k}$. From (2.12) we get that $e^{t A}\left(\operatorname{dom}\left(A^{2}\right) \cap X_{k}\right) \subseteq X_{k}$. By [20, Chapter I, Theorem 2.7], $\overline{\operatorname{dom}\left(A^{2}\right)}=X$ which implies $\overline{\operatorname{dom}\left(A^{2}\right) \cap X_{k}}=X_{k}$, therefore, $e^{t A} X_{k} \subseteq X_{k}$.

\section{I STABILITY PROPERTIES OF STANDING WAVES}

In this section we study stability/instability of the standing waves $e^{i \omega t} \boldsymbol{\varphi}_{k, \omega}^{\alpha}$, where $\boldsymbol{\varphi}_{k, \omega}^{\alpha}$ is defined by (1.5). Orbital stability is understood in the following sense. 
Definition 3.1. The standing wave $\mathbf{u}(t, x)=e^{i \omega t} \boldsymbol{\varphi ( x )}$ is said to be orbitally stable if for any $\varepsilon>0$ there exists $\eta>0$ with the following property. If $\left(\mathbf{u}_{0}, \mathbf{u}_{1}\right) \in X$ satisfies $\left\|\left(\mathbf{u}_{0}, \mathbf{u}_{1}\right)-(\varphi, i \omega \varphi)\right\|_{X}<\eta$, then the solution $\mathbf{U}(t)$ of (2.10) with $\mathbf{U}(0)=\left(\mathbf{u}_{0}, \mathbf{u}_{1}\right)$ exists globally, and

$$
\sup _{t \in[0, \infty)} \inf _{\theta \in \mathbb{R}}\left\|\mathbf{U}(t)-e^{i \theta}(\boldsymbol{\varphi}, i \omega \boldsymbol{\varphi})\right\|_{X}<\varepsilon .
$$

Otherwise, the standing wave $\mathbf{u}(t, x)=e^{i \omega t} \boldsymbol{\varphi ( x )}$ is said to be orbitally unstable.

In the sequel we will use the notation $\boldsymbol{\Phi}_{k, \omega}^{\alpha}=\left(\boldsymbol{\varphi}_{k, \omega}^{\alpha}, i \omega \boldsymbol{\varphi}_{k, \omega}^{\alpha}\right)$.

\subsection{Stability approach}

Below we will introduce basic ingredients of the classical theory by [12, 13] (see also [11, 25]). The key object is the Lyapunov functional $S_{\omega} \in C^{2}(X, \mathbb{R})$ defined by

$$
S_{\omega}(\mathbf{u}, \mathbf{v})=E(\mathbf{u}, \mathbf{v})+\omega Q(\mathbf{u}, \mathbf{v}) .
$$

From (2.9) one concludes that $\boldsymbol{\Phi}_{k, \omega}^{\alpha}$ is a critical point of $S_{\omega}$. Let $\mathcal{R}: X \rightarrow X^{*}$ be the Riesz isomorphism. A principal role in the stability/instability study is played by the spectral properties of the operator $\mathcal{R}^{-1} S_{\omega}^{\prime \prime}\left(\boldsymbol{\Phi}_{k, \omega}^{\alpha}\right): X \rightarrow X$. In what follows we will denote $\mathcal{L}_{k}^{\alpha}:=\mathcal{R}^{-1} S_{\omega}^{\prime \prime}\left(\boldsymbol{\Phi}_{k, \omega}^{\alpha}\right)$. Since $S_{\omega}^{\prime \prime}\left(\boldsymbol{\Phi}_{k, \omega}^{\alpha}\right): X \rightarrow X^{*}$ is bounded and symmetric, the operator $\mathcal{L}_{k}^{\alpha}: X \rightarrow X$ is bounded and self-adjoint, i.e.

$$
\left\langle\mathcal{L}_{k}^{\alpha} \mathbf{U}, \mathbf{V}\right\rangle_{X}=\left\langle S_{\omega}^{\prime \prime}\left(\boldsymbol{\Phi}_{k, \omega}^{\alpha}\right) \mathbf{U}, \mathbf{V}\right\rangle_{X^{*} \times X}=\left\langle S_{\omega}^{\prime \prime}\left(\boldsymbol{\Phi}_{k, \omega}^{\alpha}\right) \mathbf{V}, \mathbf{U}\right\rangle_{X^{*} \times X}=\left\langle\mathbf{U}, \mathcal{L}_{k}^{\alpha} \mathbf{V}\right\rangle_{X}, \quad \mathbf{U}, \mathbf{V} \in X
$$

Above we also have used the fact that $X$ is a real Hilbert space.

We consider the following list of assumptions about the spectrum of $\mathcal{L}_{k}^{\alpha}$ :

(A1) $n\left(\mathcal{L}_{k}^{\alpha}\right)=1$

(A2) $n\left(\mathcal{L}_{k}^{\alpha}\right)=2$

(A3) $\operatorname{ker}\left(\mathcal{L}_{k}^{\alpha}\right)=\operatorname{span}\left\{i \boldsymbol{\Phi}_{k, \omega}^{\alpha}\right\}$;

(A4) apart from the non-positive eigenvalues, $\sigma\left(\mathcal{L}_{k}^{\alpha}\right)$ is positive and bounded away from zero.

We also define the notion of linear instability.

Definition 3.2. The standing wave $e^{i \omega t} \boldsymbol{\varphi}_{k, \omega}^{\alpha}$ is linearly unstable if $\mathbf{0}$ is a linearly unstable solution for the linearized equation

$$
\mathcal{J} \partial_{t} \mathbf{V}(t)=S_{\omega}^{\prime \prime}\left(\mathbf{\Phi}_{k, \omega}^{\alpha}\right) \mathbf{V}(t)
$$

in the sense of Lyapunov.

Due to [12, 13], one can formulate the following stability/istability result.

Theorem 3.3. Let assumptions (A3), (A4) be valid, then the following two assertions hold.

(i) Suppose that $\left.\partial_{\omega} Q\left(\boldsymbol{\Phi}_{k, \omega}^{\alpha}\right)\right|_{\omega=\omega_{0}}>0$.

- If, in addition, the assumption ( $A 1)$ holds, then the standing wave $e^{i \omega_{0} t} \boldsymbol{\varphi}_{k, \omega_{0}}^{\alpha}$ is orbitally stable.

- If, in addition, the assumption (A2) holds, then the standing wave $e^{i \omega_{0} t} \boldsymbol{\varphi}_{k, \omega_{0}}^{\alpha}$ is linearly unstable.

(ii) Suppose that $\left.\partial_{\omega} Q\left(\boldsymbol{\Phi}_{k, \omega}^{\alpha}\right)\right|_{\omega=\omega_{0}}<0$ and (A1) holds, then the standing wave $e^{i \omega_{0} t} \boldsymbol{\varphi}_{k, \omega_{0}}^{\alpha}$ is orbitally unstable.

It is standard to verify that for $(\mathbf{u}, \mathbf{v}) \in X$

$$
S_{\omega}^{\prime \prime}\left(\boldsymbol{\Phi}_{k, \omega}^{\alpha}\right)(\mathbf{u}, \mathbf{v})=\left(\widetilde{H}_{\alpha} \mathbf{u}+m^{2} \mathbf{u}-\left(\boldsymbol{\varphi}_{k, \omega}^{\alpha}\right)^{p-1} \mathbf{u}-(p-1)\left(\boldsymbol{\varphi}_{k, \omega}^{\alpha}\right)^{p-1} \operatorname{Re}(\mathbf{u})+i \omega \mathbf{v}, \mathbf{v}-i \omega \mathbf{u}\right) .
$$

Here the operator $\widetilde{H}_{\alpha}$ is understood in the following sense: since bilinear form $t_{\alpha}\left(\mathbf{u}_{1}, \mathbf{u}_{2}\right)=\left\langle\mathbf{u}_{1}^{\prime}, \mathbf{u}_{2}^{\prime}\right\rangle_{L^{2}(\Gamma)}+\alpha \operatorname{Re}\left(u_{11}(0) \overline{u_{12}}(0)\right)$ is bounded on $\mathcal{E}(\Gamma)$, there exists a unique bounded operator $\widetilde{H}_{\alpha}: \mathcal{E}(\Gamma) \rightarrow \mathcal{E}^{*}(\Gamma)$ such that $t_{\alpha}\left(\mathbf{u}_{1}, \mathbf{u}_{2}\right)=\left\langle\widetilde{H}_{\alpha} \mathbf{u}_{1}, \mathbf{u}_{2}\right\rangle_{\mathcal{E}(\Gamma)^{*} \times \mathcal{E}(\Gamma)}$. 
Commonly in stability study one substitutes the operator $\mathcal{L}_{k}^{\alpha}$ acting on $X$ by the self-adjoint operator acting in $L^{2}(\Gamma) \times L^{2}(\Gamma)$ (with the real inner product). Namely, this operator is associated (by the Representation Theorem [16, Chapter VI, Theorem 2.1]) with the closed, densely defined, bounded from below bilinear form

$$
\begin{aligned}
& b_{k}(\mathbf{U}, \mathbf{V})=\left\langle S_{\omega}^{\prime \prime}\left(\boldsymbol{\Phi}_{k, \omega}^{\alpha}\right) \mathbf{U}, \mathbf{V}\right\rangle_{X^{*} \times X} \\
& =\operatorname{Re}\left[\alpha u_{1}(0) \overline{w_{1}(0)}+\int_{\Gamma}\left(\mathbf{u}^{\prime} \overline{\mathbf{w}}^{\prime}+m^{2} \mathbf{u} \overline{\mathbf{w}}-\left(\boldsymbol{\varphi}_{k, \omega}^{\alpha}\right)^{p-1} \mathbf{u} \overline{\mathbf{w}}-(p-1)\left(\boldsymbol{\varphi}_{k, \omega}^{\alpha}\right)^{p-1} \operatorname{Re}(\mathbf{u}) \overline{\mathbf{w}}\right) d x\right] \\
& +\operatorname{Re}\left[\int_{\Gamma} \mathbf{v} \overline{\mathbf{z}} d x+\omega \int_{\Gamma}(i \mathbf{v} \overline{\mathbf{w}}-i \mathbf{u} \overline{\mathbf{z}}) d x\right], \quad \mathbf{U}=(\mathbf{u}, \mathbf{v}), \mathbf{V}=(\mathbf{w}, \mathbf{z}) \in X .
\end{aligned}
$$

Proposition 3.4. The self-adjoint operator associated in $L^{2}(\Gamma) \times L^{2}(\Gamma)$ with the bilinear form $b_{k}(\mathbf{U}, \mathbf{V})$ is given by

$$
\begin{aligned}
& L_{k}^{\alpha}(\mathbf{u}, \mathbf{v})=\left(H_{\alpha} \mathbf{u}+m^{2} \mathbf{u}-\left(\boldsymbol{\varphi}_{k, \omega}^{\alpha}\right)^{p-1} \mathbf{u}-(p-1)\left(\varphi_{k, \omega}^{\alpha}\right)^{p-1} \operatorname{Re}(\mathbf{u})+i \omega \mathbf{v}, \mathbf{v}-i \omega \mathbf{u}\right), \\
& \operatorname{dom}\left(L_{k}^{\alpha}\right)=\operatorname{dom}\left(H_{\alpha}\right) \times L^{2}(\Gamma) .
\end{aligned}
$$

Moreover, $\operatorname{ker}\left(\mathcal{L}_{k}^{\alpha}\right)=\operatorname{ker}\left(L_{k}^{\alpha}\right), n\left(\mathcal{L}_{k}^{\alpha}\right)=n\left(L_{k}^{\alpha}\right)$, and

$$
\inf \sigma_{\text {ess }}\left(L_{k}^{\alpha}\right)>0 \Rightarrow \inf \sigma_{\text {ess }}\left(\mathcal{L}_{k}^{\alpha}\right)>0 .
$$

Proof. Denote by $\mathcal{T}_{k}$ the self-adjoint operator associated with the bilinear form $b_{k}(\mathbf{U}, \mathbf{V})$. Then

$$
\begin{aligned}
& \operatorname{dom}\left(\mathcal{T}_{k}\right)=\left\{\mathbf{U} \in X: \exists \mathbf{W} \in L^{2}(\Gamma) \times L^{2}(\Gamma) \text { s.t. } \forall \mathbf{V} \in X, b_{k}(\mathbf{U}, \mathbf{V})=\langle\mathbf{W}, \mathbf{V}\rangle_{L^{2}(\Gamma) \times L^{2}(\Gamma)}\right\}, \\
& \mathcal{T}_{k} \mathbf{U}=\mathbf{W} .
\end{aligned}
$$

It is easily seen that $\operatorname{dom}\left(L_{k}^{\alpha}\right) \subseteq \operatorname{dom}\left(\mathcal{T}_{k}\right)$ and $L_{k}^{\alpha} \mathbf{U}=\mathcal{T}_{k} \mathbf{U}$ for $\mathbf{U} \in \operatorname{dom}\left(L_{k}^{\alpha}\right)$. Indeed, $b_{k}(\mathbf{U}, \mathbf{V})=\left\langle L_{k}^{\alpha} \mathbf{U}, \mathbf{V}\right\rangle_{L^{2}(\Gamma) \times L^{2}(\Gamma)}$ for all $\mathbf{U} \in \operatorname{dom}\left(L_{k}^{\alpha}\right)$ and $\mathbf{V} \in X$. We need to show that $\operatorname{dom}\left(\mathcal{T}_{k}\right) \subseteq \operatorname{dom}\left(L_{k}^{\alpha}\right)$. Let $\hat{\mathbf{U}}=(\hat{\mathbf{u}}, \hat{\mathbf{v}})=\left(\left(\hat{u}_{j}\right)_{j=1}^{N},\left(\hat{v}_{j}\right)_{j=1}^{N}\right) \in \operatorname{dom}\left(\mathcal{T}_{k}\right)$. Observe that, by the definition of $\operatorname{dom}\left(\mathcal{T}_{k}\right)$, the functional $f_{k}(\mathbf{V})=b_{k}(\hat{\mathbf{U}}, \mathbf{V})=\langle\hat{\mathbf{W}}, \mathbf{V}\rangle_{L^{2}(\Gamma) \times L^{2}(\Gamma)}$ is linear and continuous on $L^{2}(\Gamma) \times L^{2}(\Gamma)$. Given $\mathbf{V}=(\mathbf{w}, \mathbf{0})$ with $\mathbf{w}=\left(w_{j}\right)_{j=1}^{N} \in C_{0}^{\infty}(\Gamma)=\bigoplus_{j=1}^{N} C_{0}^{\infty}\left(\mathbb{R}_{+}\right)$, then integrating by parts in (3.1) and using continuity of $f_{k}$, one gets that $\hat{\mathbf{u}} \in H^{2}(\Gamma)$. Finally, observing $\hat{\mathbf{u}} \in H^{2}(\Gamma)$, integrating by parts in 3.1 with $\mathbf{w} \in \mathcal{E}(\Gamma)$ such that $w_{1}(0) \neq 0$, and using continuity of $f_{k}$ again, we arrive at the conclusion that

$$
g_{k}(\mathbf{V})=\operatorname{Re}\left[\left(\alpha \hat{u}_{1}(0)-\sum_{j=1}^{N} \hat{u}_{j}^{\prime}(0)\right) \overline{w_{1}(0)}\right]
$$

has to be a continuous functional on $L^{2}(\Gamma) \times L^{2}(\Gamma)$. This is true only if $\alpha \hat{u}_{1}(0)-\sum_{j=1}^{N} \hat{u}_{j}^{\prime}(0)=0$, therefore, $\hat{\mathbf{u}} \in \operatorname{dom}\left(H_{\alpha}\right)$ and $\hat{\mathbf{U}} \in \operatorname{dom}\left(L_{k}^{\alpha}\right)$.

The second part of the proposition follows by [25, Lemma 5.4]. To apply Lemma 5.4, we only need to prove that inequality $(G)$ (Gårding's-type inequality) holds, that is, there exist $\varepsilon, C>0$ such that

$$
\left\langle S_{\omega}^{\prime \prime}\left(\boldsymbol{\Phi}_{k, \omega}^{\alpha}\right) \mathbf{V}, \mathbf{V}\right\rangle_{X^{*} \times X} \geq \varepsilon\|\mathbf{V}\|_{X}^{2}-C\|\mathbf{V}\|_{L^{2}(\Gamma) \times L^{2}(\Gamma)}^{2}, \quad \mathbf{V}=(\mathbf{w}, \mathbf{z}) \in X .
$$

From (3.1) we get

$$
\begin{aligned}
\left\langle S_{\omega}^{\prime \prime}\left(\boldsymbol{\Phi}_{k, \omega}^{\alpha}\right) \mathbf{V}, \mathbf{V}\right\rangle_{X^{*} \times X} & =\alpha\left|w_{1}(0)\right|^{2}+\int_{\Gamma}\left(\left|\mathbf{w}^{\prime}\right|^{2}+m^{2}|\mathbf{w}|^{2}-\left(\boldsymbol{\varphi}_{k, \omega}^{\alpha}\right)^{p-1}|\mathbf{w}|^{2}-(p-1)\left(\boldsymbol{\varphi}_{k, \omega}^{\alpha}\right)^{p-1}(\operatorname{Re} \mathbf{w})^{2}\right) d x+\int_{\Gamma}|\mathbf{z}|^{2} d x \\
& -2 \omega \int_{\Gamma} \operatorname{Im}(\mathbf{z} \overline{\mathbf{w}}) d x
\end{aligned}
$$

Moreover, by (2.5), we deduce

$$
\alpha\left|w_{1}(0)\right|^{2}+\frac{1}{2} \int_{\Gamma}\left|\mathbf{w}^{\prime}\right|^{2} d x=\frac{1}{2}\left(2 \alpha\left|w_{1}(0)\right|^{2}+\int_{\Gamma}\left|\mathbf{w}^{\prime}\right|^{2} d x\right) \geq \frac{-2 \alpha^{2}}{N^{2}}\|\mathbf{w}\|_{L^{2}(\Gamma)} .
$$


Denoting $M=\left\|\varphi_{k, \omega}^{\alpha}\right\|_{L^{\infty}(\Gamma)}$, we obtain from (3.3)

$$
\begin{aligned}
& \left\langle S_{\omega}^{\prime \prime}\left(\mathbf{\Phi}_{k, \omega}^{\alpha}\right) \mathbf{V}, \mathbf{V}\right\rangle_{X^{*} \times X} \geq \int_{\Gamma}\left(\frac{1}{2}\left|\mathbf{w}^{\prime}\right|^{2}+m^{2}|\mathbf{w}|^{2}+|\mathbf{z}|^{2}\right) d x-\int_{\Gamma}\left(p M^{p-1}|\mathbf{w}|^{2}-2 \omega|\mathbf{z} \| \mathbf{w}|\right) d x-\frac{2 \alpha^{2}}{N^{2}}\|\mathbf{w}\|_{L^{2}(\Gamma)} \\
& \geq \min \left\{\frac{1}{2}, m^{2}\right\}\left(\|\mathbf{w}\|_{H^{1}(\Gamma)}^{2}+\|\mathbf{z}\|_{L^{2}(\Gamma)}^{2}\right)-\left(p M^{p-1}+\frac{2 \alpha^{2}}{N^{2}}+|\omega|\right)\left(\|\mathbf{w}\|_{L^{2}(\Gamma)}^{2}+\|\mathbf{z}\|_{L^{2}(\Gamma)}^{2}\right),
\end{aligned}
$$

and therefore $(3.2$ ) holds.

\subsection{Spectral properties of $L_{k}^{\alpha}$}

Another standard step in the stability study is to express the operator $L_{k}^{\alpha}$ in more convenient form using two operators acting on real-valued functions. Let $\mathbf{U}=(\mathbf{u}, \mathbf{v}) \in \operatorname{dom}\left(L_{k}^{\alpha}\right)$ and $\mathbf{u}=\mathbf{u}_{1}+i \mathbf{u}_{2}, \mathbf{v}=\mathbf{v}_{1}+i \mathbf{v}_{2}$ with real-valued vector functions $\mathbf{u}_{j}, \mathbf{v}_{j}, j \in\{1,2\}$. We have

$$
\begin{aligned}
& L_{k}^{\alpha}(\mathbf{u}, \mathbf{v})=\left(\begin{array}{c}
-\frac{d^{2}}{d x^{2}}+m^{2}-\left(\boldsymbol{\varphi}_{k, \omega}^{\alpha}\right)^{p-1}-(p-1)\left(\boldsymbol{\varphi}_{k, \omega}^{\alpha}\right)^{p-1} \operatorname{Re}(\cdot) i \omega \\
-i \omega
\end{array}\right)\left(\begin{array}{c}
\mathbf{u}_{1}+i \mathbf{u}_{2} \\
\mathbf{v}_{1}+i \mathbf{v}_{2}
\end{array}\right) \\
& =\left(\begin{array}{c}
\left(-\mathbf{u}_{1}^{\prime \prime}+m^{2} \mathbf{u}_{1}-p\left(\boldsymbol{\varphi}_{k, \omega}^{\alpha}\right)^{p-1} \mathbf{u}_{1}-\omega \mathbf{v}_{2}\right)+i\left(-\mathbf{u}_{2}^{\prime \prime}+m^{2} \mathbf{u}_{2}-\left(\boldsymbol{\varphi}_{k, \omega}^{\alpha}\right)^{p-1} \mathbf{u}_{2}+\omega \mathbf{v}_{1}\right) \\
\left(\omega \mathbf{u}_{2}+\mathbf{v}_{1}\right)+i\left(-\omega \mathbf{u}_{1}+\mathbf{v}_{2}\right)
\end{array}\right) .
\end{aligned}
$$

Substituting the complex-valued vector function $\mathbf{U}=(\mathbf{u}, \mathbf{v})$ by the corresponding quadruplet of real-valued functions $\left(\mathbf{u}_{1}, \mathbf{u}_{2}, \mathbf{v}_{1}, \mathbf{v}_{2}\right)$, and substituting $L_{k}^{\alpha}(\mathbf{u}, \mathbf{v})=(\mathbf{f}, \mathbf{g})=\left(\mathbf{f}_{1}+i \mathbf{f}_{2}, \mathbf{g}_{1}+i \mathbf{g}_{2}\right) \in L^{2}(\Gamma) \times L^{2}(\Gamma)$ by the quadruplet $\left(\mathbf{f}_{1}, \mathbf{f}_{2}, \mathbf{g}_{1}, \mathbf{g}_{2}\right)$, we can interpret the operator $L_{k}^{\alpha}$ as

$$
\left(\begin{array}{l}
\mathbf{f}_{1} \\
\mathbf{f}_{2} \\
\mathbf{g}_{1} \\
\mathbf{g}_{2}
\end{array}\right)=\left(\begin{array}{cccc}
L_{1, k}^{\alpha}+\omega^{2} & 0 & 0 & -\omega \\
0 & L_{2, k}^{\alpha}+\omega^{2} & \omega & 0 \\
0 & \omega & 1 & 0 \\
-\omega & 0 & 0 & 1
\end{array}\right)\left(\begin{array}{l}
\mathbf{u}_{1} \\
\mathbf{u}_{2} \\
\mathbf{v}_{1} \\
\mathbf{v}_{2}
\end{array}\right)
$$

where

$$
\begin{aligned}
& L_{1, k}^{\alpha} \mathbf{u}=-\mathbf{u}^{\prime \prime}+\left(m^{2}-\omega^{2}\right) \mathbf{u}-p\left(\boldsymbol{\varphi}_{k, \omega}^{\alpha}\right)^{p-1} \mathbf{u}, \\
& L_{2, k}^{\alpha} \mathbf{u}=-\mathbf{u}^{\prime \prime}+\left(m^{2}-\omega^{2}\right) \mathbf{u}-\left(\boldsymbol{\varphi}_{k, \omega}^{\alpha}\right)^{p-1} \mathbf{u}, \quad \operatorname{dom}\left(L_{j, k}^{\alpha}\right)=\operatorname{dom}\left(H_{\alpha}\right), j \in\{1,2\} .
\end{aligned}
$$

Remark 3.5. From (3.4) we deduce

$$
(\mathbf{u}, \mathbf{v})=\left(\mathbf{u}_{1}+i \mathbf{u}_{2}, \mathbf{v}_{1}+i \mathbf{v}_{2}\right) \in \operatorname{ker}\left(L_{k}^{\alpha}\right) \Longleftrightarrow\left\{\begin{array}{l}
\mathbf{u}_{1} \in \operatorname{ker}\left(L_{1, k}^{\alpha}\right), \\
\mathbf{u}_{2} \in \operatorname{ker}\left(L_{2, k}^{\alpha}\right), \\
\mathbf{v}_{1}=-\omega \mathbf{u}_{2}, \\
\mathbf{v}_{2}=\omega \mathbf{u}_{1} .
\end{array}\right.
$$

Assuming that the operators $L_{1, k}^{\alpha}$ and $L_{2, k}^{\alpha}$ act as usual on $L^{2}(\Gamma, \mathbb{C})$ with the usual complex structure, one can prove the following result on the relation between their spectra and the spectrum of $L_{k}^{\alpha}$.

Proposition 3.6. Let $\lambda \in \mathbb{R} \backslash\{1\}$ and $\mu(\lambda):=\lambda+\frac{\lambda \omega^{2}}{1-\lambda}$. Assume also that $k \in\left\{0, \ldots,\left[\frac{N-1}{2}\right]\right\}$ and $m^{2}-\omega^{2}>\frac{\alpha^{2}}{(N-2 k)^{2}}$. Then the following assertions hold.

(i) $\lambda \in \sigma\left(L_{k}^{\alpha}\right) \Longleftrightarrow \mu(\lambda) \in \sigma\left(L_{1, k}^{\alpha}\right) \cup \sigma\left(L_{2, k}^{\alpha}\right)$.

(ii) $\operatorname{dim}\left(\operatorname{ker}\left(L_{k}^{\alpha}-\lambda\right)\right)=\operatorname{dim}\left(\operatorname{ker}\left(L_{1, k}^{\alpha}-\mu(\lambda)\right)\right)+\operatorname{dim}\left(\operatorname{ker}\left(L_{2, k}^{\alpha}-\mu(\lambda)\right)\right)$, consequently $n\left(L_{k}^{\alpha}\right)=n\left(L_{1, k}^{\alpha}\right)+n\left(L_{2, k}^{\alpha}\right)$.

(iii) $\lambda \in \sigma_{\mathrm{ess}}\left(L_{k}^{\alpha}\right) \Longleftrightarrow \mu(\lambda) \in \sigma_{\mathrm{ess}}\left(L_{1, k}^{\alpha}\right) \cup \sigma_{\mathrm{ess}}\left(L_{2, k}^{\alpha}\right)$.

(iv) Let $\mu_{-}(\lambda)$ and $\mu_{+}(\lambda)$ be the restrictions of $\mu(\lambda)$ to $(-\infty, 1)$ and $(1, \infty)$ respectively. Then

$$
\sigma_{\text {ess }}\left(L_{k}^{\alpha}\right) \backslash\{1\}=\mu_{-}^{-1}\left(\sigma_{\text {ess }}\left(L_{1, k}^{\alpha}\right) \cup \sigma_{\text {ess }}\left(L_{2, k}^{\alpha}\right)\right) \cup \mu_{+}^{-1}\left(\sigma_{\text {ess }}\left(L_{1, k}^{\alpha}\right) \cup \sigma_{\text {ess }}\left(L_{2, k}^{\alpha}\right)\right) .
$$

(v) $\sigma_{\mathrm{ess}}\left(L_{1, k}^{\alpha}\right) \cup \sigma_{\mathrm{ess}}\left(L_{2, k}^{\alpha}\right)=\sigma_{\mathrm{ess}}\left(L_{1, k}^{\alpha}\right)=\sigma_{\mathrm{ess}}\left(L_{2, k}^{\alpha}\right)=\left[m^{2}-\omega^{2}, \infty\right)$. 
(vi) $\sigma_{\text {ess }}\left(L_{k}^{\alpha}\right)=\left[\sigma_{1}, 1\right] \cup\left[\sigma_{2}, \infty\right)$, where $\sigma_{1}=\mu_{-}^{-1}\left(m^{2}-\omega^{2}\right) \in(0,1)$ and $\sigma_{2}=\mu_{+}^{-1}\left(m^{2}-\omega^{2}\right) \in(1, \infty)$.

Proof. The proof of items (i) - (iii) repeats the proof of [9, Proposition 4.5] (one just needs to substitute the operator $\mathcal{L}_{\beta}$ by $L_{k}^{\alpha}$, the operator $L_{\beta}^{-}$by $L_{2, k}^{\alpha}$, and the operator $L_{\beta}^{+}$by $L_{1, k}^{\alpha}$ ). The key point is that for $\lambda \neq 1$,

$$
\begin{aligned}
\left(L_{k}^{\alpha}-\lambda\right)(\mathbf{u}, \mathbf{v})=(\mathbf{f}, \mathbf{g}) & \Longleftrightarrow\left\{\begin{array}{r}
\left(L_{1, k}^{\alpha}+\omega^{2}-\lambda\right) \mathbf{u}_{1}-\omega \mathbf{v}_{2}=\mathbf{f}_{1}, \\
\left(L_{2, k}^{\alpha}+\omega^{2}-\lambda\right) \mathbf{u}_{2}+\omega \mathbf{v}_{1}=\mathbf{f}_{2}, \\
(1-\lambda) \mathbf{v}_{1}+\omega \mathbf{u}_{2}=\mathbf{g}_{1}, \\
(1-\lambda) \mathbf{v}_{2}-\omega \mathbf{u}_{2}=\mathbf{g}_{2} .
\end{array}\right. \\
& \left\{\begin{array}{r}
\left(L_{1, k}^{\alpha}-\mu(\lambda)\right) \mathbf{u}_{1}=\mathbf{f}_{1}+\frac{\omega}{1-\lambda} \mathbf{g}_{2}, \\
\left(L_{2, k}^{\alpha}-\mu(\lambda)\right) \mathbf{u}_{2}=\mathbf{f}_{2}-\frac{\omega}{1-\lambda} \mathbf{g}_{1}, \\
\mathbf{v}_{1}=\frac{1}{1-\lambda}\left(\mathbf{g}_{1}-\omega \mathbf{u}_{2}\right), \\
\mathbf{v}_{2}=\frac{1}{1-\lambda}\left(\mathbf{g}_{2}+\omega \mathbf{u}_{1}\right) .
\end{array}\right.
\end{aligned}
$$

Item (iv) follows from (iii) and the fact that $\mu_{-}$and $\mu_{+}$are increasing bijections.

Item $(v)$ seems natural, but we didn't manage to find its proof in the literature. Firstly, consider the self-adjoint operator

$$
\begin{aligned}
& \left(H_{\infty} \mathbf{v}\right)(x)=\left(-v_{j}^{\prime \prime}(x)\right)_{j=1}^{N}, \quad x>0, \quad \mathbf{v}=\left(v_{j}\right)_{j=1}^{N}, \\
& \operatorname{dom}\left(H_{\infty}\right)=\left\{\mathbf{v} \in H^{2}(\Gamma): v_{1}(0)=\ldots=v_{N}(0)=0\right\} .
\end{aligned}
$$

Observe that $H_{\infty}=\bigoplus_{j=1}^{N} h_{\infty}$, where

$$
\left(h_{\infty} v\right)(x)=-v^{\prime \prime}(x), \quad x>0, \quad \operatorname{dom}\left(h_{\infty}\right)=\left\{v \in H^{2}\left(\mathbb{R}_{+}\right): v(0)=0\right\} .
$$

Therefore, $\sigma_{\text {ess }}\left(H_{\infty}\right)=\sigma_{\text {ess }}\left(h_{\infty}\right)=[0, \infty)$. Secondly, notice that the operator $H_{\alpha}$ defined by (1.2) and the operator $H_{\infty}$ are self-adjoint extensions of the symmetric operator

$$
\begin{aligned}
& \left(\tilde{H}_{0} \mathbf{v}\right)(x)=\left(-v_{j}^{\prime \prime}(x)\right)_{j=1}^{N}, \quad x>0, \quad \mathbf{v}=\left(v_{j}\right)_{j=1}^{N}, \\
& \operatorname{dom}\left(\tilde{H}_{0}\right)=\left\{\mathbf{v} \in H^{2}(\Gamma): v_{1}(0)=\ldots=v_{N}(0)=0, \sum_{j=1}^{N} v_{j}^{\prime}(0)=0\right\} .
\end{aligned}
$$

The operator has equal deficiency indices $n_{+}\left(\tilde{H}_{0}\right)=1$ (see [4, proof of Theorem 3.5-(iii)]), therefore, by Krein's resolvent formula, the operator $\left(H_{\alpha}-\lambda\right)^{-1}-\left(H_{\infty}-\lambda\right)^{-1}, \lambda \in \rho\left(H_{\infty}\right) \cap \rho\left(H_{\alpha}\right)$, is of rank one (see [2, Appendix A, Theorem A.2]). Then, by Weyl's theorem [21, Theorem XIII.14], $\sigma_{\text {ess }}\left(H_{\alpha}\right)=\sigma_{\text {ess }}\left(H_{\infty}\right)=[0, \infty)$, and consequently $\sigma_{\text {ess }}\left(H_{\alpha}+m^{2}-\omega^{2}\right)=\left[m^{2}-\omega^{2}, \infty\right)$. The operator of multiplication by $\left(\boldsymbol{\varphi}_{k, \omega}^{\alpha}\right)^{p-1}$ is relatively $\left(H_{\alpha}+m^{2}-\omega^{2}\right)$-compact (for the idea of the proof see, for instance, [22, Proposition 8.20]). Therefore, $\sigma_{\mathrm{ess}}\left(L_{1, k}^{\alpha}\right)=\sigma_{\mathrm{ess}}\left(L_{2, k}^{\alpha}\right)=\left[m^{2}-\omega^{2}, \infty\right)$ (see Corollary 2 of [21, Theorem XIII.14]).

Finally, by $(v), \sigma_{\text {ess }}\left(L_{1, k}^{\alpha}\right) \cup \sigma_{\text {ess }}\left(L_{2, k}^{\alpha}\right)$ contains a neighborhood of $+\infty$. Since $\sigma_{\text {ess }}\left(L_{k}^{\alpha}\right)$ is closed, (3.5) yields (vi).

Remark 3.7. The equality $\sigma_{\mathrm{ess}}\left(H_{\alpha}\right)=[0, \infty)$ might be shown using classical Weyl's criterion (see [22, Proposition 8.11]). Let $H_{0}$ be defined by (2.4). It is easily seen that $H_{0} \geq 0$, therefore, $\sigma_{\text {ess }}\left(H_{0}\right) \subseteq \sigma\left(H_{0}\right) \subseteq[0, \infty)$. We can prove that $[0, \infty) \subseteq \sigma_{\text {ess }}\left(H_{0}\right)$.

Given $\lambda>0$. Then, by Weyl's criterion, $\lambda \in \sigma_{\text {ess }}\left(H_{0}\right)$ if, and only if, there exists a sequence $\left\{\mathbf{x}_{n}\right\}_{n=1}^{\infty} \subset \operatorname{dom}\left(H_{0}\right)$ such that

$$
\lim _{n \rightarrow \infty} \frac{\left\|\left(H_{0}-\lambda\right) \mathbf{x}_{n}\right\|_{L^{2}(\Gamma)}}{\left\|\mathbf{x}_{n}\right\|_{L^{2}(\Gamma)}}=0,
$$

and $\mathbf{x}_{n} /\left\|\mathbf{x}_{n}\right\|_{L^{2}(\Gamma)}$ tends weakly to $\mathbf{0}$ in $L^{2}(\Gamma)$.

We fix a function $\phi(x) \in C_{0}^{\infty}\left(\mathbb{R}_{+}\right)$such that

$$
\phi(x) \geq 0, \quad \phi(x)=1 \text { for } 1 / 4 \leq x \leq 1 / 2, \quad \text { and } \quad \phi(x)=0 \text { for } x \geq 1 .
$$

We set

$$
\phi_{n}(x)=\phi\left(\frac{1}{\sqrt{n}}\left(x-n^{2}\right)\right), \quad n \in \mathbb{N} .
$$


Then

It is easily seen that

$$
\operatorname{supp} \phi_{n} \subseteq\left(n^{2}, n^{2}+\sqrt{n}\right) \text {, and } \operatorname{supp} \phi_{k} \cap \operatorname{supp} \phi_{j}=\emptyset, \text { for } k \neq j, k, j \in \mathbb{N}
$$

$$
\mathbf{x}_{n}=\left(e^{i \sqrt{\lambda} x} \phi_{n}(x), \underset{2}{0}, \ldots, \underset{\mathbf{N}}{0}\right)
$$

serves for (3.6). Hence $\lambda \in \sigma_{\text {ess }}\left(H_{0}\right)$ and $\sigma_{\text {ess }}\left(H_{0}\right)=[0, \infty)$ by closedness of the essential spectrum. Then, by Weyl's theorem [21, Theorem XIII.14], $\sigma_{\text {ess }}\left(H_{\alpha}\right)=\sigma_{\text {ess }}\left(H_{0}\right)=[0, \infty)$.

\subsection{Spectral properties of $L_{1, k}^{\alpha}$ and $L_{2, k}^{\alpha}$}

Proposition 3.8. Let $\alpha \neq 0, k \in\left\{0, \ldots,\left[\frac{N-1}{2}\right]\right\}$, and $m^{2}-\omega^{2}>\frac{\alpha^{2}}{(N-2 k)^{2}}$. Then

(i) $\operatorname{ker}\left(L_{2, k}^{\alpha}\right)=\operatorname{span}\left\{\varphi_{k, \omega}^{\alpha}\right\}$ and $L_{2, k}^{\alpha} \geq 0$;

(ii) $\operatorname{ker}\left(L_{1, k}^{\alpha}\right)=\{\mathbf{0}\}$

(iii) $\operatorname{ker}\left(L_{k}^{\alpha}\right)=\left\{i \boldsymbol{\Phi}_{k, \omega}^{\alpha}\right\}$.

Proof. For the proof of (i), (ii) see [4, Proposition 1] (with $\omega$ substituted by $m^{2}-\omega^{2}$ ). The proof of (iii) follows from (i), (ii), and Remark 3.5 .

The description of the negative spectrum of $L_{1, k}^{\alpha}$ might be obtained as in [4, Theorem 3.4] and [3, Proposition 3.17]. For the reader's convenience we provide the principal steps of the proofs.

Consider the following self-adjoint Schrödinger operator on $L^{2}(\Gamma)$ with the Kirchhoff condition at $\nu=0$,

$$
\begin{aligned}
& L_{1}^{0} \mathbf{v}=-\mathbf{v}^{\prime \prime}+\left(m^{2}-\omega^{2}\right) \mathbf{v}-p \varphi_{0}^{p-1} \mathbf{v}, \\
& \operatorname{dom}\left(L_{1}^{0}\right)=\left\{\mathbf{v} \in H^{2}(\Gamma): v_{1}(0)=\ldots=v_{N}(0), \sum_{j=1}^{N} v_{j}^{\prime}(0)=0\right\},
\end{aligned}
$$

where $\varphi_{0}$ is the half-soliton solution for the classical NLS model,

$$
\varphi_{0}(x)=\left[\frac{(p+1)\left(m^{2}-\omega^{2}\right)}{2} \operatorname{sech}^{2}\left(\frac{(p-1) \sqrt{m^{2}-\omega^{2}}}{2} x\right)\right]^{\frac{1}{p-1}}
$$

From the definition of the profiles $\varphi_{k, \omega}^{\alpha}$ in 1.5 one gets

$$
\boldsymbol{\varphi}_{k, \omega}^{\alpha} \underset{\alpha \rightarrow 0}{\rightarrow} \boldsymbol{\varphi}_{0} \text { in } H^{1}(\Gamma)
$$

where $\varphi_{0}=\left(\varphi_{0}\right)_{j=1}^{N}$. To study negative spectrum of $L_{1, k}^{\alpha}$, we apply the analytic perturbation theory. Hence first we need to describe spectral properties of $L_{1}^{0}$ (which is the limiting value of $L_{1, k}^{\alpha}$ as $\alpha \rightarrow 0$ ).

Theorem 3.9. Let $L_{1}^{0}$ be defined by (3.7) and $k \in\left\{1, \ldots,\left[\frac{N-1}{2}\right]\right\}$. Then

(i) $\operatorname{ker}\left(L_{1}^{0}\right)=\operatorname{span}\left\{\hat{\boldsymbol{\varphi}}_{0,1}, \ldots, \hat{\boldsymbol{\varphi}}_{0, N-1}\right\}$, where

$$
\hat{\boldsymbol{\varphi}}_{0, j}=\left(0, \ldots, 0, \underset{\mathbf{j}}{\varphi_{0}^{\prime}}, \underset{\mathbf{j}+\mathbf{1}}{-\varphi_{0}^{\prime}}, 0, \ldots, 0\right)
$$

(ii) in the space $L_{k}^{2}(\Gamma)$ we have $\operatorname{ker}\left(L_{1}^{0}\right)=\operatorname{span}\left\{\boldsymbol{\varphi}_{0, k}\right\}$, i.e. $\operatorname{ker}\left(\left.L_{1}^{0}\right|_{L_{k}^{2}(\Gamma)}\right)=\operatorname{span}\left\{\boldsymbol{\varphi}_{0, k}\right\}$, where

$$
\boldsymbol{\varphi}_{0, k}=\left(\frac{N-k}{{ }_{\mathbf{1}}} \varphi_{0}^{\prime}, \ldots, \frac{N-k}{{ }_{\mathbf{k}}} \varphi_{0}^{\prime}, \underset{\mathbf{k}+\mathbf{1}}{-\varphi_{0}^{\prime}}, \ldots, \underset{\mathbf{N}}{-\varphi_{0}^{\prime}}\right)
$$

(iii) $n\left(L_{1}^{0}\right)=n\left(\left.L_{1}^{0}\right|_{L_{k}^{2}(\Gamma)}\right)=1$;

(iv) the rest of the spectrum of $L_{1}^{0}$ is positive and bounded away from zero. 
Proof. For the proof of $(i)-($ iii $)$ see [4, Theorem 3.5]. As in the proof of Proposition 3.6- $(v)$, one might show that $\sigma_{\mathrm{ess}}\left(L_{1}^{0}\right)=$ $\left[m^{2}-\omega^{2}, \infty\right)$ and therefore (iv) holds.

One of the principal facts for the investigation of the negative spectrum of the operator $L_{1, k}^{\alpha}$ is the following lemma.

Lemma 3.10. Let $k \in\left\{1, \ldots,\left[\frac{N-1}{2}\right]\right\}$. As a function of $\alpha,\left(L_{1, k}^{\alpha}\right)$ is a real-analytic family of self-adjoint operators of type (B) in the sense of Kato.

The above lemma and Theorem 3.9 lead to the next result.

Proposition 3.11. Let $k \in\left\{1, \ldots,\left[\frac{N-1}{2}\right]\right\}$. Then there exist $\alpha_{0}>0$ and two analytic functions $\lambda_{k}:\left(-\alpha_{0}, \alpha_{0}\right) \rightarrow \mathbb{R}$ and $\mathbf{f}_{k}:\left(-\alpha_{0}, \alpha_{0}\right) \rightarrow L_{k}^{2}(\Gamma)$ such that

(i) $\lambda_{k}(0)=0$ and $\mathbf{f}_{k}(0)=\varphi_{0, k}$, where $\varphi_{0, k}$ is defined by $(3.8)$;

(ii) for all $\alpha \in\left(-\alpha_{0}, \alpha_{0}\right), \lambda_{k}(\alpha)$ is the simple isolated second eigenvalue of $L_{1, k}^{\alpha}$ in $L_{k}^{2}(\Gamma)$, and $\mathbf{f}_{k}(\alpha)$ is the associated eigenvector for $\lambda_{k}(\alpha)$;

(iii) $\alpha_{0}$ can be chosen small enough to ensure that for $\alpha \in\left(-\alpha_{0}, \alpha_{0}\right)$ the spectrum of $L_{1, k}^{\alpha}$ in $L_{k}^{2}(\Gamma)$ is positive, except at most the first two eigenvalues.

Proof. It is implied by the Kato-Rellich theorem (see [21, Theorem XII.8]). For the details see the proof of [4, Proposition 2].

The next proposition provides characterization of $n\left(\left.L_{1, k}^{\alpha}\right|_{L_{k}^{2}(\Gamma)}\right)$ for small $\alpha$.

Proposition 3.12. Let $k \in\left\{1, \ldots,\left[\frac{N-1}{2}\right]\right\}$. There exists $0<\alpha_{1}<\alpha_{0}$ such that $\lambda_{k}(\alpha)<0$ for any $\alpha \in\left(-\alpha_{1}, 0\right)$, and $\lambda_{k}(\alpha)>0$ for any $\alpha \in\left(0, \alpha_{1}\right)$. Therefore, $n\left(\left.L_{1, k}^{\alpha}\right|_{L_{k}^{2}(\Gamma)}\right)=2$ for $\alpha<0$, and $n\left(\left.L_{1, k}^{\alpha}\right|_{L_{k}^{2}(\Gamma)}\right)=1$ for $\alpha>0$ if $\alpha$ is small enough.

Proof. From Taylor's theorem we have the expansions

$$
\lambda_{k}(\alpha)=\lambda_{0, k} \alpha+O\left(\alpha^{2}\right) \quad \text { and } \quad \mathbf{f}_{k}(\alpha)=\varphi_{0, k}+\alpha \mathbf{f}_{0, k}+\mathbf{O}\left(\alpha^{2}\right),
$$

where $\lambda_{0, k}=\lambda_{k}^{\prime}(0) \in \mathbb{R}$ and $\mathbf{f}_{0, k}=\left.\partial_{\alpha} \mathbf{f}_{k}(\alpha)\right|_{\alpha=0} \in L_{k}^{2}(\Gamma)$. The desired result will follow if we show that $\lambda_{0, k}>0$. We compute $\left\langle L_{1, k}^{\alpha} \mathbf{f}_{k}(\alpha), \boldsymbol{\varphi}_{0, k}\right\rangle_{L^{2}(\Gamma)}$ in two different ways.

Note that for $\varphi_{k, \omega}^{\alpha}$ defined by (1.5) we have

$$
\begin{gathered}
\boldsymbol{\varphi}_{k, \omega}^{\alpha}=\boldsymbol{\varphi}_{0}+\alpha \mathbf{g}_{0, k}+\mathbf{O}\left(\alpha^{2}\right), \\
\mathbf{g}_{0, k}=\left.\partial_{\alpha} \boldsymbol{\varphi}_{k, \omega}^{\alpha}\right|_{\alpha=0}=\frac{2}{(p-1)(N-2 k)\left(m^{2}-\omega^{2}\right)}\left(\begin{array}{c}
\varphi_{0}^{\prime}, \ldots, \varphi_{0}^{\prime},-\varphi_{0}^{\prime}, \ldots,-\varphi_{0}^{\prime} \\
\mathbf{k}+\mathbf{N}
\end{array}\right) .
\end{gathered}
$$

From (3.9) we obtain

$$
\left\langle L_{1, k}^{\alpha} \mathbf{f}_{k}(\alpha), \varphi_{0, k}\right\rangle_{L^{2}(\Gamma)}=\lambda_{0, k} \alpha\left\|\varphi_{0, k}\right\|_{L^{2}(\Gamma)}^{2}+O\left(\alpha^{2}\right)
$$

By $L_{1}^{0} \varphi_{0, k}=\mathbf{0}$ and $(3.9)$ we get

$$
L_{1, k}^{\alpha} \boldsymbol{\varphi}_{0, k}=p\left(\left(\boldsymbol{\varphi}_{0}\right)^{p-1}-\left(\boldsymbol{\varphi}_{k, \omega}^{\alpha}\right)^{p-1}\right) \boldsymbol{\varphi}_{0, k}=-\alpha p(p-1)\left(\boldsymbol{\varphi}_{0}\right)^{p-2} \mathbf{g}_{0, k} \boldsymbol{\varphi}_{0, k}+\mathbf{O}\left(\alpha^{2}\right)
$$

The operations in the last equality are componentwise. Equations $\underline{3.12}$, (3.10), and $\varphi_{0, k} \in \operatorname{dom}\left(H_{\alpha}\right)$ lead to

$$
\begin{aligned}
& \left\langle L_{1, k}^{\alpha} \mathbf{f}_{k}(\alpha), \boldsymbol{\varphi}_{0, k}\right\rangle_{L^{2}(\Gamma)}=\left\langle\mathbf{f}_{k}(\alpha), L_{1, k}^{\alpha} \boldsymbol{\varphi}_{0, k}\right\rangle_{L^{2}(\Gamma)}=-\left\langle\boldsymbol{\varphi}_{0, k}, \alpha p(p-1)\left(\boldsymbol{\varphi}_{0}\right)^{p-2} \mathbf{g}_{0, k} \boldsymbol{\varphi}_{0, k}\right\rangle_{L^{2}(\Gamma)}+O\left(\alpha^{2}\right) \\
& =-\alpha p(p-1)\left(\frac{2}{k}-(N-k)\right) \frac{2}{(p-1)(N-2 k)\left(m^{2}-\omega^{2}\right)} \int_{0}^{\infty}\left(\varphi_{0}^{\prime}\right)^{3} \varphi_{0}^{p-2} d x+O\left(\alpha^{2}\right) \\
& =-2 \alpha p \frac{N-k}{k\left(m^{2}-\omega^{2}\right)} \int_{0}^{\infty}\left(\varphi_{0}^{\prime}\right)^{3} \varphi_{0}^{p-2} d x+O\left(\alpha^{2}\right) .
\end{aligned}
$$


Finally, combining (3.13) and 3.11, we obtain

$$
\lambda_{0, k}=\frac{-2 p \frac{N-k}{k\left(m^{2}-\omega^{2}\right)} \int_{0}^{\infty}\left(\varphi_{0}^{\prime}\right)^{3} \varphi_{0}^{p-2} d x}{\left\|\varphi_{0, k}\right\|_{L^{2}(\Gamma)}^{2}}+O(\alpha) .
$$

It follows that $\lambda_{0, k}$ is positive for sufficiently small $|\alpha|$ (due to the negativity of $\varphi_{0}^{\prime}$ on $\mathbb{R}_{+}$), which in view of (3.9) ends the proof.

Summarizing the above results, we obtain the following characterization of the negative spectrum of $L_{1, k}^{\alpha}$.

Proposition 3.13. (i) Let $k \in\left\{1, \ldots,\left[\frac{N-1}{2}\right]\right\}$.

(a) If $\alpha>0$, then $n\left(\left.L_{1, k}^{\alpha}\right|_{L_{k}^{2}(\Gamma)}\right)=1$.

(b) If $\alpha<0$, then $n\left(\left.L_{1, k}^{\alpha}\right|_{L_{k}^{2}(\Gamma)}\right)=2$.

(ii) Let $k=0$.

(a) If $\alpha<0$, then $n\left(L_{1,0}^{\alpha}\right)=1$ in $L^{2}(\Gamma)$.

(b) If $\alpha>0$, then $n\left(\left.L_{1,0}^{\alpha}\right|_{L_{\mathrm{eq}}^{2}(\Gamma)}\right)=1$.

Proof. (i) The proof of the proposition is analogous to the one of [4, Proposition 4]. It uses Proposition 3.12 and a classical continuation argument based on the Riesz projection.

(ii) (a) Observe that the operator $L_{1,0}^{\alpha}$ is the self-adjoint extension of the non-negative symmetric operator

$$
\begin{aligned}
& L_{0} \mathbf{v}=L_{1,0}^{\alpha} \mathbf{v}, \quad \mathbf{v} \in \operatorname{dom}\left(L_{0}\right), \\
& \operatorname{dom}\left(L_{0}\right)=\left\{\mathbf{v} \in H^{2}(\Gamma): v_{1}(0)=\ldots=v_{N}(0)=0, \sum_{j=1}^{N} v_{j}^{\prime}(0)=0\right\},
\end{aligned}
$$

with deficiency indices $n_{ \pm}\left(L_{0}\right)=1$ (see the proof of [3, Theorem 3.12-(iii)]). Hence $n\left(L_{1,0}^{\alpha}\right) \leq 1$ by [17, §14, Theorem 16]. Since $\left\langle L_{1,0}^{\alpha} \varphi_{0, \omega}^{\alpha}, \varphi_{0, \omega}^{\alpha}\right\rangle_{L^{2}(\Gamma)}=-(p-1)\left\|\varphi_{0, \omega}^{\alpha}\right\|_{L^{p+1}(\Gamma)}^{p+1}<0$, we get the equality $n\left(L_{1,0}^{\alpha}\right)=1$.

(b) Consider the restriction $\left.L_{1,0}^{\alpha}\right|_{L_{\mathrm{eq}}^{2}(\Gamma)}$ as the self-adjoint extension of the following symmetric operator

$$
\begin{aligned}
& \tilde{L}_{0} \mathbf{v}=L_{1,0}^{\alpha} \mathbf{v}, \quad \mathbf{v} \in \operatorname{dom}\left(\tilde{L}_{0}\right), \\
& \operatorname{dom}\left(\tilde{L}_{0}\right)=\left\{\mathbf{v} \in \operatorname{dom}\left(H_{\alpha}\right) \cap L_{\mathrm{eq}}^{2}(\Gamma): v_{1}\left(b_{0}\right)=\ldots=v_{N}\left(b_{0}\right)=0\right\},
\end{aligned}
$$

where $b_{0}=-\frac{2}{(p-1) \sqrt{m^{2}-\omega^{2}}} c_{0}$, and $c_{0}$ is defined in $(1.5)$. Let us prove that $\tilde{L}_{0}$ is non-negative. Observe that every component of the vector $\mathbf{v}=\left(v_{j}\right)_{j=1}^{N} \in H^{2}(\Gamma)$ satisfies the identity

$$
-v_{j}^{\prime \prime}+\omega v_{j}-p\left(\tilde{\varphi}_{0, j}\right)^{p-1} v_{j}=\frac{-1}{\tilde{\varphi}_{0, j}^{\prime}} \frac{d}{d x}\left[\left(\tilde{\varphi}_{0, j}^{\prime}\right)^{2} \frac{d}{d x}\left(\frac{v_{j}}{\tilde{\varphi}_{0, j}^{\prime}}\right)\right], x \in \mathbb{R}_{+} \backslash\left\{b_{0}\right\} .
$$

Using the above equality and integrating by parts, we get for $\mathbf{v} \in \operatorname{dom}\left(\tilde{L}_{0}\right)$,

$$
\begin{aligned}
& \left\langle\tilde{L}_{0} \mathbf{v}, \mathbf{v}\right\rangle_{L_{\mathrm{eq}}^{2}(\Gamma)}=N\left(\int_{0}^{b_{0}-}+\int_{b_{0}+}^{+\infty}\right)\left(\tilde{\varphi}_{0,1}^{\prime}\right)^{2}\left|\frac{d}{d x}\left(\frac{v_{1}}{\tilde{\varphi}_{0,1}^{\prime}}\right)\right|^{2} d x+N\left[-v_{1}^{\prime} \bar{v}_{1}+\left|v_{1}\right|^{2} \frac{\tilde{\varphi}_{0,1}^{\prime \prime}}{\tilde{\varphi}_{0,1}^{\prime}}\right]_{0}^{\infty} \\
& +N\left[v_{1}^{\prime} \bar{v}_{1}-\left|v_{1}\right|^{2} \frac{\tilde{\varphi}_{0,1}^{\prime \prime}}{\tilde{\varphi}_{0,1}^{\prime}}\right]_{b_{0}-}^{b_{0}+}=N\left(\int_{0}^{b_{0}-}+\int_{b_{0}+}^{+\infty}\right)\left(\tilde{\varphi}_{0,1}^{\prime}\right)^{2}\left|\frac{d}{d x}\left(\frac{v_{1}}{\tilde{\varphi}_{0,1}^{\prime}}\right)\right|^{2} d x \geq 0 .
\end{aligned}
$$

The deficiency indices of $\tilde{L}_{0}$ are

$$
n_{ \pm}\left(\tilde{L}_{0}\right)=\operatorname{dim}\left(\operatorname{dom}\left(\left.L_{1,0}^{\alpha}\right|_{L_{\text {eq }}^{2}(\Gamma)}\right)\right)-\operatorname{dim}\left(\operatorname{dom}\left(\tilde{L}_{0}\right)\right)=1 .
$$

Therefore, $n\left(\left.L_{1,0}^{\alpha}\right|_{L_{\text {eq }}^{2}(\Gamma)}\right) \leq 1$ by [17, §14, Theorem 16]. One also has that $\varphi_{0, \omega}^{\alpha} \in L_{\text {eq }}^{2}(\Gamma)$ and $\left\langle L_{1,0}^{\alpha} \varphi_{0, \omega}^{\alpha}, \varphi_{0, \omega}^{\alpha}\right\rangle_{L^{2}(\Gamma)}=-(p-$ 1) $\left\|\varphi_{0, \omega}^{\alpha}\right\|_{L^{p+1}(\Gamma)}^{p+1}<0$. Hence $n\left(\left.L_{1,0}^{\alpha}\right|_{L_{\mathrm{eq}}^{2}(\Gamma)}\right)=1$. 
Remark 3.14. In [3], we considered the NLS equation with the $\delta$-interaction on the star graph $\Gamma$. In particular, we proved [3, Theorem 1.1] on the orbital instability of the profile $\boldsymbol{\Phi}_{\alpha, \delta}=\boldsymbol{\varphi}_{0 \text {, 俩 }}^{\alpha}$ (where $m^{2}-\omega^{2}$ has to be substituted by $\omega$ ). Using the proof of item $(i i)-(b)$, we may complete the result of Theorem 1.1 in [3]. Indeed, one may deduce analogously that $n\left(\left.\mathbf{L}_{1, \alpha}\right|_{L^{2}(\Gamma)}\right)=1$, where the operator $\mathbf{L}_{1, \alpha}$ is defined in [3, Subsection 3.1]. Using Proposition 3.17 and Proposition 3.19 (ii) - 2), 3) in [3], we can affirm for $\alpha>0$ the following two results.

(i) Let $3<p<5$, then there exists $\omega_{2}>\frac{\alpha^{2}}{N^{2}}$ such that $e^{i \omega t} \boldsymbol{\Phi}_{\alpha, \delta}$ is orbitally unstable in $\mathcal{E}_{\text {eq }}(\Gamma)$ and therefore in $\mathcal{E}(\Gamma)$ for $\omega \in\left(\frac{\alpha^{2}}{N^{2}}, \omega_{2}\right)$. Moreover, $e^{i \omega t} \boldsymbol{\Phi}_{\alpha, \delta}$ is orbitally stable in $\mathcal{E}_{\mathrm{eq}}(\Gamma)$ for $\omega>\omega_{2}$.

(ii) Let $p \geq 5$, then $e^{i \omega t} \boldsymbol{\Phi}_{\alpha, \delta}$ is orbitally unstable in $\mathcal{E}_{\mathrm{eq}}(\Gamma)$, and therefore in $\mathcal{E}(\Gamma)$ for $\omega>\frac{\alpha^{2}}{N^{2}}$.

Remark 3.15. Using the approach of [15] (see Theorem 3.1), one may show that in $L^{2}(\Gamma)$,

$$
n\left(L_{1, k}^{\alpha}\right)=\left\{\begin{array}{c}
k+1, \text { for } \alpha<0, \\
N-k, \text { for } \alpha>0,
\end{array} \quad k \in\left\{0, \ldots,\left[\frac{N-1}{2}\right]\right\} .\right.
$$

\subsection{Slope condition}

Let $k \in\left\{0, \ldots,\left[\frac{N-1}{2}\right]\right\}$. In this subsection we study the sign of $\partial_{\omega} Q\left(\boldsymbol{\Phi}_{k, \omega}^{\alpha}\right)$. By the definition of $Q$ and $\boldsymbol{\Phi}_{k, \omega}^{\alpha}=\left(\boldsymbol{\varphi}_{k, \omega}^{\alpha}, i \omega \boldsymbol{\varphi}_{k, \omega}^{\alpha}\right)$, we get (see [4, Proposition 5])

$$
Q\left(\boldsymbol{\Phi}_{k, \omega}^{\alpha}\right)=-\omega\left\|\varphi_{k, \omega}^{\alpha}\right\|_{L^{2}(\Gamma)}^{2}=Q_{k, 1}(\omega) Q_{k, 2}(\omega)
$$

where

$$
\begin{gathered}
Q_{k, 1}(\omega)=-2 \omega\left(\frac{p+1}{2}\right)^{\frac{2}{p-1}} \frac{\left(m^{2}-\omega^{2}\right)^{\frac{5-p}{2(p-1)}}}{p-1}, \\
Q_{k, 2}(\omega)=\int_{\frac{-\alpha}{(2 k-N) \sqrt{m^{2}-\omega^{2}}}}^{1} k\left(1-t^{2}\right)^{\frac{3-p}{p-1}} d t+\int_{\frac{\alpha}{(2 k-N) \sqrt{m^{2}-\omega^{2}}}}^{1}(N-k)\left(1-t^{2}\right)^{\frac{3-p}{p-1}} d t .
\end{gathered}
$$

Using the above formulas for $Q\left(\Phi_{k, \omega}^{\alpha}\right)$, we obtain the result.

Lemma 3.16. Let $1<p<5$.

(i) $\partial_{\omega} Q\left(\boldsymbol{\Phi}_{k, \omega}^{\alpha}\right)>0$ for

$$
\alpha<0, \quad \text { and } \quad \omega \in\left(-m, \frac{-m \sqrt{p-1}}{2}\right) \cup\left(\frac{m \sqrt{p-1}}{2}, m\right) .
$$

(ii) $\partial_{\omega} Q\left(\boldsymbol{\Phi}_{k, \omega}^{\alpha}\right)<0$ for

$$
\alpha>0, \quad \text { and } \quad \omega \in\left(\frac{-m \sqrt{p-1}}{2}, 0\right) \cup\left(0, \frac{m \sqrt{p-1}}{2}\right) .
$$

Proof. By (3.14), $\partial_{\omega} Q\left(\boldsymbol{\Phi}_{k, \omega}^{\alpha}\right)=Q_{k, 1}^{\prime}(\omega) Q_{k, 2}(\omega)+Q_{k, 1}(\omega) Q_{k, 2}^{\prime}(\omega)$. From 3.15) we get

$$
\begin{aligned}
& Q_{k, 1}^{\prime}(\omega)=\frac{2}{p-1}\left(\frac{p+1}{2}\right)^{\frac{2}{p-1}}\left(m^{2}-\omega^{2}\right)^{\frac{7-3 p}{2(p-1)}}\left(\frac{4 \omega^{2}}{p-1}-m^{2}\right), \\
& Q_{k, 2}^{\prime}(\omega)=\left(1-\frac{\alpha^{2}}{(2 k-N)^{2}\left(m^{2}-\omega^{2}\right)}\right)^{\frac{3-p}{p-1}} \frac{\alpha \omega}{\left(m^{2}-\omega^{2}\right)^{3 / 2}} .
\end{aligned}
$$

Observe that $Q_{k, 2}(\omega)>0$ for any $\omega$ and $\alpha$. It is easily seen that

$$
\left\{\begin{array} { l } 
{ Q _ { k , 1 } ^ { \prime } ( \omega ) > 0 , } \\
{ Q _ { k , 2 } ( \omega ) > 0 , } \\
{ Q _ { k , 2 } ^ { \prime } ( \omega ) < 0 , } \\
{ Q _ { k , 1 } ( \omega ) < 0 }
\end{array} \Leftrightarrow \left\{\begin{array}{c}
\alpha<0, \\
\omega \in\left(\frac{m \sqrt{p-1}}{2}, m\right)
\end{array}\right.\right.
$$


implies $\partial_{\omega} Q\left(\boldsymbol{\Phi}_{k, \omega}^{\alpha}\right)>0$. Analogously

$$
\left\{\begin{array} { l } 
{ Q _ { k , 1 } ^ { \prime } ( \omega ) > 0 , } \\
{ Q _ { k , 2 } ( \omega ) > 0 , } \\
{ Q _ { k , 2 } ^ { \prime } ( \omega ) > 0 , } \\
{ Q _ { k , 1 } ( \omega ) > 0 }
\end{array} \Leftrightarrow \left\{\begin{array}{c}
\alpha<0, \\
\omega \in\left(-m, \frac{-m \sqrt{p-1}}{2}\right)
\end{array}\right.\right.
$$

yields $\partial_{\omega} Q\left(\boldsymbol{\Phi}_{k, \omega}^{\alpha}\right)>0$. Finally, $(i)$ holds.

To show (ii), observe that

$$
\left\{\begin{array} { l } 
{ Q _ { k , 1 } ^ { \prime } ( \omega ) < 0 , } \\
{ Q _ { k , 2 } ( \omega ) > 0 , } \\
{ Q _ { k , 2 } ^ { \prime } ( \omega ) > 0 , } \\
{ Q _ { k , 1 } ( \omega ) < 0 }
\end{array} \Leftrightarrow \left\{\begin{array}{c}
\alpha>0, \\
\omega \in\left(0, \frac{m \sqrt{p-1}}{2}\right)
\end{array}\right.\right.
$$

implies $\partial_{\omega} Q\left(\boldsymbol{\Phi}_{k, \omega}^{\alpha}\right)<0$, and

$$
\left\{\begin{array} { l } 
{ Q _ { k , 1 } ^ { \prime } ( \omega ) < 0 , } \\
{ Q _ { k , 2 } ( \omega ) > 0 , } \\
{ Q _ { k , 2 } ^ { \prime } ( \omega ) < 0 , } \\
{ Q _ { k , 1 } ( \omega ) > 0 }
\end{array} \Leftrightarrow \left\{\begin{array}{c}
\alpha>0, \\
\omega \in\left(\frac{-m \sqrt{p-1}}{2}, 0\right)
\end{array}\right.\right.
$$

yields $\partial_{\omega} Q\left(\boldsymbol{\Phi}_{k, \omega}^{\alpha}\right)<0$.

Combining Lemma 2.7, Theorem 3.3, Proposition 3.4, Proposition 3.6, Proposition 3.8, and Proposition 3.13, we get stability/instability result.

Theorem 3.17. Assume that $1<p<5$ and $m>0$.

(i) Let $k \in\left\{1, \ldots,\left[\frac{N-1}{2}\right]\right\}$ and $m^{2}-\omega^{2}>\frac{\alpha^{2}}{(N-2 k)^{2}}$.

(a) For $\alpha>0$ and $\omega \in\left(\frac{-m \sqrt{p-1}}{2}, 0\right) \cup\left(0, \frac{m \sqrt{p-1}}{2}\right)$ the standing wave $e^{i \omega t} \varphi_{k, \omega}^{\alpha}$ is orbitally unstable in $X_{k}$ and therefore in $X$.

(b) For $\alpha<0$ and $\omega \in\left(-m, \frac{-m \sqrt{p-1}}{2}\right) \cup\left(\frac{m \sqrt{p-1}}{2}, m\right)$ (if such $\omega$ exists) the standing wave $e^{i \omega t} \boldsymbol{\varphi}_{k, \omega}^{\alpha}$ is linearly unstable.

(ii) Let $k=0$ and $m^{2}-\omega^{2}>\frac{\alpha^{2}}{N^{2}}$.

(a) For $\alpha>0$ and $\omega \in\left(\frac{-m \sqrt{p-1}}{2}, 0\right) \cup\left(0, \frac{m \sqrt{p-1}}{2}\right)$ the standing wave $e^{i \omega t} \boldsymbol{\varphi}_{0, \omega}^{\alpha}$ is orbitally unstable in $X_{\text {eq }}$ and therefore in $X$.

(b) For $\alpha<0$ and $\omega \in\left(-m, \frac{-m \sqrt{p-1}}{2}\right) \cup\left(\frac{m \sqrt{p-1}}{2}, m\right)$ (if such $\omega$ exists) the standing wave $e^{i \omega t} \boldsymbol{\varphi}_{0, \omega}^{\alpha}$ is orbitally stable in $X$.

Proof. Observe that, by Proposition 3.4 Proposition 3.6-(vi), Proposition 3.8-(iii), assumptions (A3) and (A4) are satisfied.

(i) (a) By Proposition 3.13-(i), for $\alpha>0$ we have $n\left(\left.L_{1, k}^{\alpha}\right|_{L_{k}^{2}(\Gamma)}\right)=1$. If additionally $\omega \in\left(\frac{-m \sqrt{p-1}}{2}, 0\right) \cup\left(0, \frac{m \sqrt{p-1}}{2}\right)$, then $\partial_{\omega} Q\left(\boldsymbol{\Phi}_{k, \omega}^{\alpha}\right)<0$ (see Lemma 3.16-(ii)).

By Proposition 3.4, 3.8-(i) and 3.6-(ii), we obtain $n\left(\left.\mathcal{L}_{k}^{\alpha}\right|_{X_{k}}\right)=1$. Finally, by Theorem 3.3-(ii), we get orbital instability of $e^{i \omega t} \boldsymbol{\varphi}_{k, \omega}^{\alpha}$ in $X_{k}$ since (2.2) is well-posed in $X_{k}$ by Lemma 2.7. Consequently $e^{i \omega t} \boldsymbol{\varphi}_{k, \omega}^{\alpha}$ is orbitally unstable in $X$.

(b) By Proposition 3.13 $(i)$, for $\alpha<0$ we have $n\left(\left.L_{1, k}^{\alpha}\right|_{L_{k}^{2}(\Gamma)}\right)=2$ and analogously to the previous case $n\left(\left.\mathcal{L}_{k}^{\alpha}\right|_{X_{k}}\right)=2$. If additionally $\omega \in\left(-m, \frac{-m \sqrt{p-1}}{2}\right) \cup\left(\frac{m \sqrt{p-1}}{2}, m\right)$, then $\partial_{\omega} Q\left(\boldsymbol{\Phi}_{k, \omega}^{\alpha}\right)>0$ (see Lemma 3.16-(i)). Finally, by Theorem 3.3- $(i)$, we get linear instability of $e^{i \omega t} \boldsymbol{\varphi}_{k, \omega}^{\alpha}$.

(ii) (a) By Proposition 3.13 (ii), for $\alpha>0$ we have $n\left(\left.L_{1, k}^{\alpha}\right|_{L_{\text {eq }}^{2}(\Gamma)}\right)=1$, and therefore $n\left(\left.\mathcal{L}_{k}^{\alpha}\right|_{X_{\text {eq }}}\right)=1$. If additionally $\omega \in$ $\left(\frac{-m \sqrt{p-1}}{2}, 0\right) \cup\left(0, \frac{m \sqrt{p-1}}{2}\right)$, then $\partial_{\omega} Q\left(\boldsymbol{\Phi}_{0, \omega}^{\alpha}\right)<0$ (see Lemma $\left.3.16-(i i)\right)$. 
Finally, by Theorem 3.3-(ii), we get orbital instability of $e^{i \omega t} \boldsymbol{\varphi}_{0, \omega}^{\alpha}$ in $X_{\text {eq }}$ since (2.2) is well-posed in $X_{\text {eq }}$ by Lemma 2.7 Consequently $e^{i \omega t} \boldsymbol{\varphi}_{0, \omega}^{\alpha}$ is orbitally unstable in $X$.

(b) By Proposition 3.13 ( $(i)$, for $\alpha<0$ we have $n\left(L_{1, k}^{\alpha}\right)=1$ and consequently $n\left(\mathcal{L}_{k}^{\alpha}\right)=1$. If additionally $\omega \in$ $\left(-m, \frac{-m \sqrt{p-1}}{2}\right) \cup\left(\frac{m \sqrt{p-1}}{2}, m\right)$, then $\partial_{\omega} Q\left(\boldsymbol{\Phi}_{0, \omega}^{\alpha}\right)>0$ (see Lemma 3.16- $\left.(i)\right)$. By Theorem 3.3- $(i)$, we get orbital stability of $e^{i \omega t} \boldsymbol{\varphi}_{0, \omega}^{\alpha}$ in $X$.

\section{References}

[1] R. Adami, C. Cacciapuoti, D. Finco, D. Noja, Variational properties and orbital stability of standing waves for NLS equation on a star graph, J. Differential Equations 257 (2014), no. 10, 3738-3777.

[2] S. Albeverio, F. Gesztesy, R. Hoegh-Krohn, and H. Holden, Solvable Models in Quantum Mechanics, $2^{\text {nd }}$ edition, AMS Chelsea Publishing, Providence, RI, 2005.

[3] J. Angulo, N. Goloshchapova, Extension theory approach in the stability of the standing waves for the NLS equation with point interactions on a star graph, Advances in Differential Equations 23 (2018), 793-846.

[4] J. Angulo, N. Goloshchapova, On the orbital instability of excited states for the NLS equation with the $\delta$-interaction on a star graph, Discrete \& Continuous Dynamical Systems 38 (2018), no. 10, 5039-5066.

[5] J.M. Ball, Strongly continuous semigroups, weak solution and the variation of constants formula, Proc. Amer. Math. Soc. 63 (1977), 370-373.

[6] V. Banica, L.I. Ignat, Dispersion for the Schrödinger equation on the line with multiple Dirac delta potentials and on delta trees, Anal. Partial Differ. Equ. 7 (2014), no. 4, 903-927.

[7] G. Berkolaiko and P. Kuchment, Introduction to quantum graphs, Mathematical Surveys and Monographs, 186, Amer. Math. Soc., Providence, RI, 2013.

[8] C. Cacciapuoti, D. Finco, and D. Noja, Ground state and orbital stability for the NLS equation on a general starlike graph with potentials, Nonlinearity 30 (2017), 3271-3303.

[9] E. Csobo, F. Genoud, M. Ohta, J. Royer, Stability of Standing Waves for a Nonlinear Klein-Gordon Equation with Delta Potentials, arXiv:1810.04874v1.

[10] T. Cazenave, A. Haraux, An Introduction to Semilinear Evolution Equations, Oxford Lecture Series in Mathematics and its Applications 13, Oxford University Press, 1998.

[11] S. De Bièvre, F. Genoud, S. Rota-Nodari, Orbital stability: analysis meets geometry, in Nonlinear optical and atomic systems, arXiv:1407.5951v2.

[12] M. Grillakis, J. Shatah and W. Strauss, Stability theory of solitary waves in the presence of symmetry. I, J. Funct. Anal. 74 (1987), no. 1, 160-197.

[13] M. Grillakis, J. Shatah and W. Strauss, Stability theory of solitary waves in the presence of symmetry. II, J. Funct. Anal. 94 (1990), no. 2, 308-348.

[14] L. Jeanjean, S. Le Coz, Instability for standing waves of nonlinear Klein-Gordon equations via mountain-pass arguments, Trans. Amer. Math. Soc. 361 (2009), 5401-5416.

[15] A. Kairzhan, Orbital instability of standing waves for NLS equation on star graphs, Proc. Amer. Math. Soc. 147 (2019), 2911-2924.

[16] T. Kato, Perturbation Theory for Linear Operators, Die Grundlehren der Mathematischen Wissenschaften, Band 132, Springer-Verlag New York, Inc., New York, 1966.

[17] M.A. Naimark, Linear Differential Operators. Part II. Linear Differential Operators in Hilbert Space, Frederick Ungar, New York, 1968.

[18] D. Noja, Nonlinear Schrödinger equation on graphs: recent results and open problems, Philos. Trans. R. Soc. Lond. Ser. A Math. Phys. Eng. Sci. 372 (2014), 20130002, 20 pp. 
[19] M. Ohta, G. Todorova, Strong instability of standing waves for the nonlinear Klein-Gordon equation and the KleinGordon-Zakharov system, SIAM J. Math. Anal. 38 (2007), 1912-1931.

[20] A. Pazy, Semigroups of Linear Operators and Applications to Partial Differential Equations, Applied Mathematical Sciences 44, Springer-Verlag, New York, 1983.

[21] M. Reed and B. Simon, Methods of Modern Mathematical Physics. IV. Analysis of Operators, Academic Press, New York, 1978.

[22] K. Schmüdgen, Unbounded Self-adjoint Operators on Hilbert Space, Graduate Texts in Mathematics, 265, Springer, Dordrecht, 2012.

[23] J. Shatah, Stable standing waves of nonlinear Klein-Gordon equations, Comm. Math. Phys. 91 (1983), 313-327.

[24] J. Shatah, Unstable ground state of nonlinear Klein-Gordon equations, Trans. Amer. Math. Soc. 290 (1985), 701-710.

[25] C. A. Stuart, Lectures on the Orbital Stability of Standing Waves and Application to the Nonlinear Schrödinger Equation, Milan Journal of Mathematics 76 (2008), 329-399. 